\title{
Modeling Correlated Defaults: First Passage Model under Stochastic Volatility*
}

\author{
Jean-Pierre Fouque ${ }^{\dagger}$ Brian C. Wignall ${ }^{\ddagger}$ Xianwen $\mathrm{Zhou}^{\S}$
}

Submitted: July 3, 2006

Revised November 6, 2007

\begin{abstract}
Default dependency structure is crucial in pricing multi-name credit derivatives as well as in credit risk management. In this paper, we extend the first passage model for one name with stochastic volatility (Fouque-Sircar-Sølna, Applied Mathematical Finance 2006) to the multi-name case. Correlation of defaults is generated by correlation between the Brownian motions driving the individual names as well as through common stochastic volatility factors. A numerical example for the loss distribution of a portfolio of defaultable bonds is examined after stochastic volatility is incorporated.
\end{abstract}

\section{Introduction}

Default dependency structure is a crucial issue in pricing multi-name credit derivatives as well as in credit risk management. For a multi-name credit derivative, the default dependency structure among the underlying portfolio of reference entities is as important as, and in many cases even more important than, the individual term structures of default probabilities.

On the other hand, it is well documented in the finance literature that stock returns exhibit stochastic volatility. It has been shown in Fouque, Papanicolaou and Sircar (2000) that asymptotic methods are very efficient in capturing the effects of stochastic volatility in simple robust corrections to the constant volatility formulas. Recently, by incorporating stochastic volatility to the first passage model developed by Black and Cox (1976) in modeling defaultable bonds, Fouque, Sircar

\footnotetext{
*This work is based on the third author's $\mathrm{PhD}$ dissertation where the "uncorrelated" case is treated. Work supported by NSF grant DMS-0455982

${ }^{\dagger}$ Department of Statistics and Applied Probability, University of California, Santa Barbara, CA 93106-3110, fouque@pstat.ucsb.edu.

${ }^{\ddagger}$ Department of Statistics and Applied Probability, University of California, Santa Barbara, CA 93106-3110, wignall@pstat.ucsb.edu.

${ }^{\S}$ Fixed Income Division, Lehman Brothers, NY, stephenok@hotmail.com.
} 
and Sølna (2006) obtained much higher yield spreads for short maturity bonds than those under constant volatility. In fact those low yield spreads at short maturities is exactly what the original first passage model - in fact all firm's value models - have been criticized for. By using models incorporating fast and slow stochastic volatility factors, and a combination of singular and regular perturbations techniques they obtain reasonable fits to defaultable bonds data.

In this paper, we extend the first passage model to model default dependency in two directions: by extending to multi-dimension and by incorporating stochastic volatility. We derive approximations for the joint survival probabilities and subsequently for the distribution of number of defaults in a basket of names. Since we are not considering in this paper particular structured products such as CDOs, we do not specify all the features of the defaultable bonds except that an individual bond defaults at the first time that the underlying firm's value goes to or below some exogenously prespecified level - the default threshold. Also, since we are mainly interested in the number of defaults before maturity, recovery does not play a role. If we were pricing derivatives, such as tranches of CDOs, which is outside the scope of the paper, we would have to incorporate recovery. This can be done following the remark in Fouque et al. (2006) (Section 2) by considering more general boundary value conditions.

The rest of this paper is organized as follows. We first set up in Section 2 the class of models that we consider. We study first the case where the Brownian motions driving the names are independent so that the correlation of defaults is only due to the common factors of stochastic volatility. Then in Section 3, using combined regular and singular perturbation techniques, as in Fouque et al. (2003) for a single name, we obtain approximations for the joint survival probabilities. Specifically, we give a formula for the leading order term and we characterize the corrections terms as solutions of partial differential equations. The derivation of these equations is presented in Appendix A and explicit formulas are given in Appendix B. In Section 4, we examine the loss distribution for a portfolio of defaultable bonds. The general case is examined briefly, and then a special case - homogeneous portfolio case - is examined in detail. Following this, some numerical results illustrating the effect of stochastic volatility are presented for the homogeneous portfolio case. Finally in Section 5 we generalize our model to the case where the Brownian motions driving the names are correlated. We show that an expansion around the independent case can be performed and gives a tractable way to combine this source of default correlation with the one coming from stochastic volatility. Our result shows that for a given maturity the dependency generated by correlating the Brownian motions driving the names is of the same nature as the one generated by stochastic volatility. However they differ across maturities and therefore we conclude that both sources of default correlation should be taken into account when pricing CDO's tranches for instance.

\section{Model Setup}

We consider a pool of $n$ defaultable bonds whose underlying firms' value processes $\left\{X_{t}^{(i)}\right\}_{i=1}^{n}$ exhibit the following multi-factor stochastic volatility dynamics under the physical probability measure $\mathbb{P}$ - the real world probability measure:

$$
\begin{aligned}
& \mathrm{d} X_{t}^{(1)}=\mu_{1} X_{t}^{(1)} \mathrm{d} t+f_{1}\left(Y_{t}, Z_{t}\right) X_{t}^{(1)} \mathrm{d} W_{t}^{(1)}, \\
& \mathrm{d} X_{t}^{(2)}=\mu_{2} X_{t}^{(2)} \mathrm{d} t+f_{2}\left(Y_{t}, Z_{t}\right) X_{t}^{(2)} \mathrm{d} W_{t}^{(2)}
\end{aligned}
$$




$$
\begin{aligned}
\cdots \cdots & \cdots \cdots \cdots \cdots \cdots \\
\mathrm{d} X_{t}^{(n)} & =\mu_{n} X_{t}^{(n)} \mathrm{d} t+f_{n}\left(Y_{t}, Z_{t}\right) X_{t}^{(n)} \mathrm{d} W_{t}^{(n)}, \\
\mathrm{d} Y_{t} & =\frac{1}{\epsilon}\left(m_{Y}-Y_{t}\right) \mathrm{d} t+\frac{\nu_{Y} \sqrt{2}}{\sqrt{\epsilon}} \mathrm{d} W_{t}^{(Y)}, \\
\mathrm{d} Z_{t} & =\delta\left(m_{Z}-Z_{t}\right) \mathrm{d} t+\nu_{Z} \sqrt{2 \delta} \mathrm{d} W_{t}^{(Z)},
\end{aligned}
$$

where $W_{t}^{(i)}$ 's are standard Brownian motions and we consider first the uncorrelated case corresponding to $\mathrm{d}\left\langle W^{(i)}, W^{(j)}\right\rangle_{t} \equiv \rho_{i j} d t=0$ for $i \neq j$. The stochastic volatility correlation structure is given by:

$$
\mathrm{d}\left\langle W^{(Y)}, W^{(i)}\right\rangle_{t}=\rho_{i Y} \mathrm{~d} t, \quad \mathrm{~d}\left\langle W^{(Z)}, W^{(i)}\right\rangle_{t}=\rho_{i Z} \mathrm{~d} t, \quad \mathrm{~d}\left\langle W^{(Y)}, W^{(Z)}\right\rangle_{t}=\rho_{Y Z} \mathrm{~d} t
$$

with all $\rho$ 's being constant numbers between -1 and 1 . Note that $\sum_{i=1}^{n} \rho_{i Y}^{2} \leq 1$ and $\sum_{i=1}^{n} \rho_{i Z}^{2} \leq 1$ must be satisfied if the Brownian motions $W_{t}^{(i)}$ 's are to be independent. Also $\mu_{i}, \epsilon, \delta, m_{Y}, \nu_{Y}, m_{Z}, \nu_{Z}$ are all constant numbers with $\epsilon>0$ and $\delta>0$ both being small so that stochastic volatilities are driven by two Ornstein-Uhlenbeck (OU) processes, $Y_{t}$ being fast mean-reverting with rate of meanreversion $1 / \epsilon$ and the invariant distribution $\mathcal{N}\left(m_{Y}, \nu_{Y}^{2}\right)$, and $Z_{t}$ being slowly mean-reveting with rate of mean-reversion $\delta$ and the invariant distribution $\mathcal{N}\left(m_{Z}, \nu_{Z}^{2}\right)$. The function $f_{i}$ 's are positive functions, smooth with respect to the slow variable $z$, and are assumed here, for instance, to be bounded above and below away from zero. If the stochastic volatility is turned off by choosing $f_{i}=0, i=1, \ldots, n$, then the defaults become independent and the default of a given firm follows the model developed by Black and Cox (1976).

Remark. We start by assuming independence among the Brownian motions $W_{t}^{(i)}$ 's for $i=1,2, \ldots, n$ basically for two reasons. Firstly, we try to avoid the intractability caused by the interdependence, as can be seen in Zhou (2001) where results on the joint distribution of two hitting times are derived. This dependency will be restored in Section 5 and made tractable by using a perturbation argument around the uncorrelated case. Secondly, we argue that the dependence among the defaultable names introduced through stochastic volatilities is as important as the dependence generated by the interdependence among the Brownian motions driving them. This important issue will be discussed further in Section 5 .

Under the risk-neutral probability measure $\tilde{\mathbb{P}}$, chosen by the market through derivatives trading, the dynamics becomes

$$
\begin{aligned}
& \mathrm{d} X_{t}^{(1)}=r X_{t}^{(1)} \mathrm{d} t+f_{1}\left(Y_{t}, Z_{t}\right) X_{t}^{(1)} \mathrm{d} \tilde{W}_{t}^{(1)}, \\
& \mathrm{d} X_{t}^{(2)}=r X_{t}^{(2)} \mathrm{d} t+f_{2}\left(Y_{t}, Z_{t}\right) X_{t}^{(2)} \mathrm{d} \tilde{W}_{t}^{(2)}, \\
& \ldots \ldots \ldots \ldots \ldots \ldots \\
& \mathrm{d} X_{t}^{(n)}=r X_{t}^{(n)} \mathrm{d} t+f_{n}\left(Y_{t}, Z_{t}\right) X_{t}^{(n)} \mathrm{d} \tilde{W}_{t}^{(n)} \\
& \mathrm{d} Y_{t}=\left[\frac{1}{\epsilon}\left(m_{Y}-Y_{t}\right)-\frac{\nu_{Y} \sqrt{2}}{\sqrt{\epsilon}} \Lambda_{1}\left(Y_{t}, Z_{t}\right)\right] \mathrm{d} t+\frac{\nu_{Y} \sqrt{2}}{\sqrt{\epsilon}} \mathrm{d} \tilde{W}_{t}^{(Y)}, \\
& \mathrm{d} Z_{t}=\left[\delta\left(m_{Z}-Z_{t}\right)-\nu_{Z} \sqrt{2 \delta} \Lambda_{2}\left(Y_{t}, Z_{t}\right)\right] \mathrm{d} t+\nu_{Z} \sqrt{2 \delta} \mathrm{d} \tilde{W}_{t}^{(Z)},
\end{aligned}
$$

where $r$ is the riskfree interest rate (assumed constant here), $\tilde{W}_{t}^{(i)}$, s are standard Brownian motions with $\mathrm{d}\left\langle\tilde{W}^{(i)}, \tilde{W}^{(j)}\right\rangle_{t}=0$ for $i \neq j$, and

$$
\mathrm{d}\left\langle\tilde{W}^{(Y)}, \tilde{W}^{(i)}\right\rangle_{t}=\rho_{i Y} \mathrm{~d} t, \quad \mathrm{~d}\left\langle\tilde{W}^{(Z)}, \tilde{W}^{(i)}\right\rangle_{t}=\rho_{i Z} \mathrm{~d} t, \quad \mathrm{~d}\left\langle\tilde{W}^{(Y)}, \tilde{W}^{(Z)}\right\rangle_{t}=\rho_{Y Z} \mathrm{~d} t
$$


The quantity of interest are the joint survival probabilities of $n$ given firms since they are the building blocks when one wants to compute the probability distribution of the number of defaults as explained in Section 4.

For fixed time $T>0$, our objective is to find the joint (risk-neutral) survival probability

$$
u^{\epsilon, \delta}(t, \mathbf{x}, y, z) \equiv \tilde{\mathbb{P}}\left\{\tau_{t}^{(1)}>T, \ldots, \tau_{t}^{(n)}>T \mid \mathbf{X}_{t}=\mathbf{x}, Y_{t}=y, Z_{t}=z\right\},
$$

where $t<T, \mathbf{X}_{t} \equiv\left(X_{t}^{(1)}, \ldots, X_{t}^{(n)}\right), \mathbf{x} \equiv\left(x_{1}, \ldots, x_{n}\right)$, and $\tau_{t}^{(i)}$ is the default time of firm $i$, defined as follows,

$$
\tau_{t}^{(i)}=\inf \left\{s \geq t, X_{s}^{(i)} \leq B_{i}(s)\right\}
$$

where $B_{i}(t)$ is the exogenously pre-specified default threshold at time $t$ for firm $i$. Here we follow Black and Cox (1976) and we assume that

$$
B_{i}(t)=K_{i} \mathrm{e}^{\eta_{i} t},
$$

with $K_{i}>0$ and $\eta_{i} \geq 0$, all being constant numbers. It is very common that credit derivatives have long maturities. Therefore it is more realistic to assume time varying default thresholds (exponentially growing in our case) than constant ones. Observe that $u^{\epsilon, \delta}$ is zero whenever $x_{i} \leq$ $B_{i}(t)$ for some $i$ and therefore we only need to focus on the case where $x_{i}>B_{i}(t)$ for all $i=$ $1,2, \ldots, n$.

\section{Approximated Joint Survival Probabilities}

We derive in this section an approximation for the joint survival probability $u^{\epsilon, \delta}$ defined in (1). We first write a PDE representation for it and we then perform singular and regular perturbations with respect to the small parameters $\epsilon$ and $\delta$.

\subsection{PDE Representation}

In terms of partial differential equations (PDE), $u^{\epsilon, \delta}$ is the solution to the following boundary value problem:

$$
\begin{aligned}
\mathcal{L}^{\epsilon, \delta} u^{\epsilon, \delta} & =0, \quad x_{i}>B_{i}(t), \text { for all } i, t<T, \\
u^{\epsilon, \delta}\left(t, x_{1}, x_{2}, \ldots, x_{n}, y, z\right) & =0, \quad \exists i \in\{1, \cdots, n\}, x_{i}=B_{i}(t), t \leq T, \\
u^{\epsilon, \delta}\left(T, x_{1}, x_{2}, \ldots, x_{n}, y, z\right) & =1, \quad x_{i}>B_{i}(t), \text { for all } i,
\end{aligned}
$$

where the operator $\mathcal{L}^{\epsilon, \delta}$ has the following decomposition in terms of powers of $\sqrt{\epsilon}$ and $\sqrt{\delta}$ :

$$
\mathcal{L}^{\epsilon, \delta}=\frac{1}{\epsilon} \mathcal{L}_{0}+\frac{1}{\sqrt{\epsilon}} \mathcal{L}_{1}+\mathcal{L}_{2}+\sqrt{\delta} \mathcal{M}_{1}+\delta \mathcal{M}_{2}+\sqrt{\frac{\delta}{\epsilon}} \mathcal{M}_{3}
$$


with the notations

$$
\begin{aligned}
\mathcal{L}_{0} & =\left(m_{Y}-y\right) \frac{\partial}{\partial y}+\nu_{Y}^{2} \frac{\partial^{2}}{\partial y^{2}} \\
\mathcal{L}_{1} & =\nu_{Y} \sqrt{2}\left[\sum_{i=1}^{n} \rho_{i Y} f_{i}(y, z) x_{i} \frac{\partial^{2}}{\partial x_{i} \partial y}-\Lambda_{1}(y, z) \frac{\partial}{\partial y}\right], \\
\mathcal{L}_{2} & =\frac{\partial}{\partial t}+\sum_{i=1}^{n}\left(\frac{1}{2} f_{i}^{2}(y, z) x_{i}^{2} \frac{\partial^{2}}{\partial x_{i}^{2}}+r x_{i} \frac{\partial}{\partial x_{i}}\right), \\
\mathcal{M}_{1} & =\nu_{Z} \sqrt{2}\left[\sum_{i=1}^{n} \rho_{i Z} f_{i}(y, z) x_{i} \frac{\partial^{2}}{\partial x_{i} \partial z}-\Lambda_{2}(y, z) \frac{\partial}{\partial z}\right] \\
\mathcal{M}_{2} & =\left(m_{Z}-z\right) \frac{\partial}{\partial z}+\nu_{Z}^{2} \frac{\partial^{2}}{\partial z^{2}} \\
\mathcal{M}_{3} & =2 \rho_{Y} \nu_{Y} \nu_{Z} \frac{\partial^{2}}{\partial y \partial z} .
\end{aligned}
$$

As in Fouque et al. (2003), we expand $u^{\epsilon, \delta}$ in terms of powers of $\sqrt{\epsilon}$ and $\sqrt{\delta}$ :

$$
u^{\epsilon, \delta}=u_{0}+\sqrt{\epsilon} u_{1,0}+\sqrt{\delta} u_{0,1}+\epsilon u_{2,0}+\sqrt{\epsilon \delta} u_{1,1}+\delta u_{0,2}+\cdots,
$$

and retain

$$
\tilde{u} \equiv u_{0}+\sqrt{\epsilon} u_{1,0}+\sqrt{\delta} u_{0,1}
$$

as our approximation for $u^{\epsilon, \delta}$. In the Appendix A we present the formal expansion argument leading to the characterization of the leading order term $u_{0}$ and the two correction terms $\sqrt{\epsilon} u_{1,0}$ and $\sqrt{\delta} u_{0,1}$. In the following sections we compute these terms in the context of our multidimensional boundary value problem.

\subsection{Leading Order Term $u_{0}$}

Following Fouque et al. (2003) as explained in the Appendix A, the leading order term $u_{0}$ is independent of $y$ and is determined by the following PDE system with respect to the variables $(t, x)$, the variable $z$ being simply a parameter:

$$
\begin{aligned}
\left\langle\mathcal{L}_{2}\right\rangle u_{0} & =0, \quad x_{i}>B_{i}(t), \text { for all } i, t<T, \\
u_{0}\left(t, x_{1}, x_{2}, \ldots, x_{n}\right) & =0, \quad \exists i \in\{1, \cdots, n\}, x_{i}=B_{i}(t), t \leq T, \\
u_{0}\left(T, x_{1}, x_{2}, \ldots, x_{n}\right) & =1, \quad x_{i}>B_{i}(t), \text { for all } i,
\end{aligned}
$$

where, from the definition $(3)$ of $\mathcal{L}_{2}$, we have

$$
\left\langle\mathcal{L}_{2}\right\rangle=\frac{\partial}{\partial t}+\sum_{i=1}^{n}\left(\frac{1}{2}\left\langle f_{i}^{2}(\cdot, z)\right\rangle x_{i}^{2} \frac{\partial^{2}}{\partial x_{i}^{2}}+r x_{i} \frac{\partial}{\partial x_{i}}\right) .
$$


The variable $z$ appears only as a parameter in the averaged diffusion coefficients $\left\langle f_{i}^{2}(\cdot, z)\right\rangle$ with respect to the invariant distribution $\mathcal{N}\left(m_{Y}, \nu_{Y}^{2}\right)$ of the process $Y_{t}$ under the real world measure $\mathbb{P}$, i.e.,

$$
\left\langle f_{i}^{2}(\cdot, z)\right\rangle=\int f_{i}^{2}(y, z) \frac{1}{\nu_{Y} \sqrt{2 \pi}} \exp \left(-\frac{\left(y-m_{Y}\right)^{2}}{2 \nu_{Y}^{2}}\right) \mathrm{d} y
$$

Proposition 1 The leading order term $u_{0}$ in the approximation (5) is given by:

$$
u_{0}=\prod_{i=1}^{n} Q_{i} \equiv \prod_{i=1}^{n}\left[\mathrm{~N}\left(d_{2(i)}^{+}\right)-\left(\frac{x_{i}}{B_{i}(t)}\right)^{p_{i}} \mathrm{~N}\left(d_{2(i)}^{-}\right)\right],
$$

where $\mathrm{N}(\cdot)$ is the standard cumulative normal distribution function, and

$$
\begin{aligned}
d_{2(i)}^{ \pm} & \equiv \frac{ \pm \ln \frac{x_{i}}{B_{i}(t)}+\left(r-\eta_{i}-\frac{\sigma_{i}^{2}(z)}{2}\right)(T-t)}{\sigma_{i}(z) \sqrt{T-t}}, \\
\sigma_{i}(z) & \left.\equiv \sqrt{\left\langle f_{i}^{2}(\cdot, z)\right\rangle}, \quad \text { effective volatility of firm } i\right) \\
p_{i} & \equiv 1-\frac{2\left(r-\eta_{i}\right)}{\sigma_{i}^{2}(z)} .
\end{aligned}
$$

Proof From (6) and (7), $u_{0}$ admits the probabilistic representation:

$$
u_{0}=\overline{\mathbb{E}}\left\{\prod_{i=1}^{n} \mathbf{1}_{\left\{\inf _{t \leq s \leq T} \bar{X}_{s}^{(i)} / B_{i}(s)>1\right\}} \mid \overline{\mathbf{X}}_{s}=\mathbf{x}\right\},
$$

where, under the probability measure $\overline{\mathbb{P}}$, we have

$$
\begin{aligned}
& \mathrm{d} \bar{X}_{t}^{(1)}=r \bar{X}_{t}^{(1)} \mathrm{d} t+\sigma_{1}(z) \bar{X}_{t}^{(1)} \mathrm{d} \bar{W}_{t}^{(1)}, \\
& \mathrm{d} \bar{X}_{t}^{(2)}=r \bar{X}_{t}^{(2)} \mathrm{d} t+\sigma_{2}(z) \bar{X}_{t}^{(2)} \mathrm{d} \bar{W}_{t}^{(2)}, \\
& \text {....................... } \\
& \mathrm{d} \bar{X}_{t}^{(n)}=r \bar{X}_{t}^{(n)} \mathrm{d} t+\sigma_{n}(z) \bar{X}_{t}^{(n)} \mathrm{d} \bar{W}_{t}^{(n)},
\end{aligned}
$$

with $\bar{W}^{(i)}$ 's being independent standard Brownian motions. In other words, $\left\{\bar{X}_{t}^{(i)}\right\}, i=1,2, \ldots, n$, are independent Geometric Brownian motions and it follows that

$$
u_{0}=\prod_{i=1}^{n} \overline{\mathbb{P}}\left\{\inf _{t \leq s \leq T} \bar{X}_{s}^{(i)} / B_{i}(s)>1 \mid \bar{X}_{t}^{(i)}=x_{i}\right\} .
$$

By doing change of variables to make the corresponding boundary constant (instead of exponentially growing) and then using the appropriate formula in Borodin and Salminen (2002) obtained by the method of images or equivalently the reflection principle in one dimension, one can reach the conclusion in the proposition. 


\subsection{Correction Term $\sqrt{\epsilon} u_{1,0}$}

From Appendix A the term $u_{1,0}$ is determined by the following PDE system:

$$
\begin{aligned}
\left\langle\mathcal{L}_{2}\right\rangle u_{1,0} & =\mathcal{A} u_{0}, \quad x_{i}>B_{i}(t), \text { for all } i, t<T, \\
u_{1,0}\left(t, x_{1}, x_{2}, \ldots, x_{n}\right) & =0, \quad \exists i \in\{1, \cdots, n\}, x_{i}=B_{i}(t), t \leq T, \\
u_{1,0}\left(T, x_{1}, x_{2}, \ldots, x_{n}\right) & =0, \quad x_{i}>B_{i}(t), \text { for all } i
\end{aligned}
$$

where the operator $\mathcal{A}$ is given by

$$
\begin{aligned}
\mathcal{A} \equiv & \left\langle\mathcal{L}_{1} \mathcal{L}_{0}^{-1}\left(\mathcal{L}_{2}-\left\langle\mathcal{L}_{2}\right\rangle\right)\right\rangle \\
= & \frac{\nu_{Y}}{\sqrt{2}}\left[\sum_{i=1}^{n} \sum_{j=1}^{n} \rho_{i Y}\left\langle f_{i} \frac{\partial \phi_{j}}{\partial y}\right\rangle x_{i} \frac{\partial}{\partial x_{i}}\left(x_{j}^{2} \frac{\partial^{2}}{\partial x_{j}^{2}}\right)-\sum_{j=1}^{n}\left\langle\Lambda_{1} \frac{\partial \phi_{j}}{\partial y}\right\rangle x_{j}^{2} \frac{\partial^{2}}{\partial x_{j}^{2}}\right] \\
= & -\frac{\nu_{Y}}{\sqrt{2}} \sum_{i=1}^{n}\left\langle\Lambda_{1} \frac{\partial \phi_{i}}{\partial y}\right\rangle x_{i}^{2} \frac{\partial^{2}}{\partial x_{i}^{2}}+\frac{\nu_{Y}}{\sqrt{2}} \sum_{i=1}^{n} \rho_{i Y}\left\langle f_{i} \frac{\partial \phi_{i}}{\partial y}\right\rangle x_{i} \frac{\partial}{\partial x_{i}}\left(x_{i}^{2} \frac{\partial^{2}}{\partial x_{i}^{2}}\right) \\
& +\frac{\nu_{Y}}{\sqrt{2}} \sum_{\substack{i, j=1 \\
i \neq j}}^{n} \rho_{i Y}\left\langle f_{i} \frac{\partial \phi_{j}}{\partial y}\right\rangle x_{i} \frac{\partial}{\partial x_{i}}\left(x_{j}^{2} \frac{\partial^{2}}{\partial x_{j}^{2}}\right) .
\end{aligned}
$$

Here $\phi_{i}(y, z)$, for $i=1,2, \ldots, n$, denote solutions of reasonable growth at infinity to the following Poisson equations with respect to the variable $y$ :

$$
\mathcal{L}_{0} \phi_{i}(y, z)=f_{i}^{2}(y, z)-\left\langle f_{i}^{2}(\cdot, z)\right\rangle,
$$

and $\langle\cdot\rangle$ denotes the average with respect to the invariant distribution $\mathcal{N}\left(m_{Y}, \nu_{Y}^{2}\right)$ of $Y$.

The following result shows that the multi-dimensional problem (9) can be reduced to many uncoupled one- and two-dimensional problems.

Proposition 2 The correction term $\sqrt{\epsilon} u_{1,0}$ is given by

$$
\sqrt{\epsilon} u_{1,0}=\sum_{i=1}^{n} R_{i}^{(2)} w_{i}^{(2)} \prod_{\substack{j=1 \\ j \neq i}}^{n} Q_{j}+\sum_{i=1}^{n} R_{i}^{(3)} w_{i}^{(3)} \prod_{\substack{j=1 \\ j \neq i}}^{n} Q_{j}+\sum_{\substack{i, j=1 \\ i \neq j}}^{n} R_{i j}^{(3)} w_{i j}^{(3)} \prod_{\substack{k=1 \\ k \neq i, j}}^{n} Q_{k},
$$

where the coefficients $R_{i}^{(2)}, R_{i}^{(3)}, R_{i j}^{(3)}$ depend on the parameter $z$ and are given by

$$
\begin{aligned}
R_{i}^{(2)} & =-\frac{\nu_{Y} \sqrt{\epsilon}}{\sqrt{2}}\left\langle\Lambda_{1}(\cdot, z) \frac{\partial \phi_{i}}{\partial y}(\cdot, z)\right\rangle \\
R_{i}^{(3)} & =\frac{\nu_{Y} \sqrt{\epsilon}}{\sqrt{2}} \rho_{i Y}\left\langle f_{i}(\cdot, z) \frac{\partial \phi_{i}}{\partial y}(\cdot, z)\right\rangle \\
R_{i j}^{(3)} & =\frac{\nu_{Y} \sqrt{\epsilon}}{\sqrt{2}} \rho_{i Y}\left\langle f_{i}(\cdot, z) \frac{\partial \phi_{j}}{\partial y}(\cdot, z)\right\rangle, i \neq j,
\end{aligned}
$$


with $\phi_{i}$ 's given by (11) and $Q_{i}$ 's given in Proposition 1, and where the functions $w_{i}^{(2)}\left(t, x_{i}\right), w_{i}^{(3)}\left(t, x_{i}\right)$ and $w_{i j}^{(3)}\left(t, x_{i}, x_{j}\right)$ depend on the parameter $z$ and are given by the following problems:

$$
\begin{aligned}
& {\left[\frac{\partial}{\partial t}+\frac{1}{2} \sigma_{i}^{2}(z) x_{i}^{2} \frac{\partial^{2}}{\partial x_{i}^{2}}+r x_{i} \frac{\partial}{\partial x_{i}}\right] w_{i}^{(2)}=x_{i}^{2} \frac{\partial^{2} Q_{i}}{\partial x_{i}^{2}}, \quad x_{i}>B_{i}(t), t<T,} \\
& w_{i}^{(2)}\left(t, B_{i}(t)\right)=0, \quad t \leq T, \\
& w_{i}^{(2)}\left(T, x_{i}\right)=0, \quad x_{i}>B_{i}(t) \\
& {\left[\frac{\partial}{\partial t}+\frac{1}{2} \sigma_{i}^{2}(z) x_{i}^{2} \frac{\partial^{2}}{\partial x_{i}^{2}}+r x_{i} \frac{\partial}{\partial x_{i}}\right] w_{i}^{(3)}=x_{i} \frac{\partial}{\partial x_{i}}\left(x_{i}^{2} \frac{\partial^{2} Q_{i}}{\partial x_{i}^{2}}\right), \quad x_{i}>B_{i}(t), t<T,} \\
& w_{i}^{(3)}\left(t, B_{i}(t)\right)=0, \quad t \leq T, \\
& w_{i}^{(3)}\left(T, x_{i}\right)=0, \quad x_{i}>B_{i}(t), \\
& {\left[\frac{\partial}{\partial t}+\frac{1}{2} \sigma_{i}^{2}(z) x_{i}^{2} \frac{\partial^{2}}{\partial x_{i}^{2}}+\frac{1}{2} \sigma_{j}^{2}(z) x_{j}^{2} \frac{\partial^{2}}{\partial x_{j}^{2}}+r x_{i} \frac{\partial}{\partial x_{i}}+r x_{j} \frac{\partial}{\partial x_{j}}\right] w_{i j}^{(3)}=\left(x_{i} \frac{\partial Q_{i}}{\partial x_{i}}\right)\left(x_{j}^{2} \frac{\partial^{2} Q_{j}}{\partial x_{j}^{2}}\right)} \\
& x_{i}>B_{i}(t), x_{j}>B_{j}(t), t<T, \\
& w_{i j}^{(3)}\left(t, x_{i}, x_{j}\right)=0, \quad \text { if } \quad x_{i}=B_{i}(t) \quad \text { or } \quad x_{j}=B_{j}(t), t \leq T \text {, } \\
& w_{i j}^{(3)}\left(T, x_{i}, x_{j}\right)=0, \quad x_{i}>B_{i}(t), x_{j}>B_{j}(t),
\end{aligned}
$$

with $\sigma_{i}(z)$ given in Proposition 1.

Proof Note that with the form (10) of $\mathcal{A}$ and the definitions $(13,14,15)$ we have:

$$
\sqrt{\epsilon} \mathcal{A}=\sum_{i=1}^{n} R_{i}^{(2)} x_{i}^{2} \frac{\partial^{2}}{\partial x_{i}^{2}}+\sum_{i=1}^{n} R_{i}^{(3)} x_{i} \frac{\partial}{\partial x_{i}}\left(x_{i}^{2} \frac{\partial^{2}}{\partial x_{i}^{2}}\right)+\sum_{\substack{i, j=1 \\ i \neq j}}^{n} R_{i j}^{(3)} x_{i} \frac{\partial}{\partial x_{i}}\left(x_{j}^{2} \frac{\partial^{2}}{\partial x_{j}^{2}}\right) .
$$

By linearity of (9), it is enough to check that

$$
\begin{aligned}
\left\langle\mathcal{L}_{2}\right\rangle\left(w_{i}^{(2)} \prod_{j=1, j \neq i}^{n} Q_{j}\right) & =x_{i}^{2} \frac{\partial^{2} u_{0}}{\partial x_{i}^{2}}, \\
\left\langle\mathcal{L}_{2}\right\rangle\left(w_{i}^{(3)} \prod_{j=1, j \neq i}^{n} Q_{j}\right) & =x_{i} \frac{\partial}{\partial x_{i}}\left(x_{i}^{2} \frac{\partial^{2} u_{0}}{\partial x_{i}^{2}}\right), \\
\left\langle\mathcal{L}_{2}\right\rangle\left(w_{i j}^{(3)} \prod_{k=1, k \neq i, j}^{n} Q_{k}\right) & =x_{i} \frac{\partial}{\partial x_{i}}\left(x_{j}^{2} \frac{\partial^{2} u_{0}}{\partial x_{j}^{2}}\right) .
\end{aligned}
$$

Using the form (7) of $\left\langle\mathcal{L}_{2}\right\rangle$ and $u_{0}=\prod_{i=1}^{n} Q_{i}$ one can easily check that (19), (20), and (21) are satisfied. The boundary and terminal conditions for the correction $\sqrt{\epsilon} u_{1,0}$ are directly inherited from the boundary and terminal conditions for the functions $w_{i}^{(2)}$ ' $\mathrm{s}, w_{i}^{(3)}$ ' $\mathrm{s}, w_{i j}^{(3)}$ 's, and $Q_{i}$ 's.

The problems (16), (17) and (18) are boundary value problems with sources. In Appendix B we show how to transform them into boundary value problems without source leading to explicit formulas (up to Gaussian integrals). 


\subsection{Correction Term $\sqrt{\delta} u_{0,1}$}

From Appendix A the term $u_{0,1}$ is determined by the following PDE system:

$$
\begin{aligned}
\left\langle\mathcal{L}_{2}\right\rangle u_{0,1} & =-\left\langle\mathcal{M}_{1}\right\rangle u_{0}, \quad x_{i}>B_{i}(t), \text { for all } i, t<T, \\
u_{0,1}\left(t, x_{1}, x_{2}, \ldots, x_{n}\right) & =0, \quad \exists i \in\{1, \cdots, n\}, x_{i}=B_{i}(t), t \leq T, \\
u_{0,1}\left(T, x_{1}, x_{2}, \ldots, x_{n}\right) & =0, \quad x_{i}>B_{i}(t), \text { for all } i .
\end{aligned}
$$

where the operator $\left\langle\mathcal{M}_{1}\right\rangle$ is given by:

$$
\left\langle\mathcal{M}_{1}\right\rangle=\nu_{Z} \sqrt{2}\left[\sum_{i=1}^{n} \rho_{i Z}\left\langle f_{i}(\cdot, z)\right\rangle x_{i} \frac{\partial^{2}}{\partial x_{i} \partial z}-\left\langle\Lambda_{2}(\cdot, z)\right\rangle \frac{\partial}{\partial z}\right] .
$$

The following result shows that, as for $u_{1,0}$, the multi-dimensional problem $(22)$ for $u_{0,1}$ can be reduced to many uncoupled one- and two-dimensional problems.

Proposition 3 The correction term $\sqrt{\delta} u_{0,1}$ is given by

$$
\sqrt{\delta} u_{0,1}=\sum_{i=1}^{n} R_{i}^{(0)} w_{i}^{(0)} \prod_{\substack{j=1 \\ j \neq i}}^{n} Q_{j}+\sum_{i=1}^{n} R_{i}^{(1)} w_{i}^{(1)} \prod_{\substack{j=1 \\ j \neq i}}^{n} Q_{j}+\sum_{\substack{i, j=1 \\ i \neq j}}^{n} R_{i j}^{(1)} w_{i j}^{(1)} \prod_{\substack{k=1 \\ k \neq i, j}}^{n} Q_{k},
$$

where the coefficients $R_{i}^{(0)}, R_{i}^{(1)}, R_{i j}^{(1)}$ depend on the parameter $z$ and are given by

$$
\begin{aligned}
& R_{i}^{(0)}=-\nu_{Z} \sqrt{2 \delta}\left\langle\Lambda_{2}(\cdot, z)\right\rangle \sigma_{i}^{\prime}(z) \\
& R_{i}^{(1)}=\nu_{Z} \sqrt{2 \delta} \rho_{i Z}\left\langle f_{i}(\cdot, z)\right\rangle \sigma_{i}^{\prime}(z) \\
& R_{i j}^{(1)}=\nu_{Z} \sqrt{2 \delta} \rho_{i Z}\left\langle f_{i}(\cdot, z)\right\rangle \sigma_{j}^{\prime}(z), \quad i \neq j,
\end{aligned}
$$

$\sigma_{i}(z)$ 's and $Q_{i}$ 's given in Proposition $1, \sigma_{i}^{\prime}=d \sigma_{i} / d z$, and where the functions $w_{i}^{(0)}\left(t, x_{i} ; z\right), w_{i}^{(1)}\left(t, x_{i} ; z\right)$ and $w_{i j}^{(1)}\left(t, x_{i}, x_{j} ; z\right)$ depend on the parameter $z$ and are given by the following problems:

$$
\begin{aligned}
{\left[\frac{\partial}{\partial t}+\frac{1}{2} \sigma_{i}^{2}(z) x_{i}^{2} \frac{\partial^{2}}{\partial x_{i}^{2}}+r x_{i} \frac{\partial}{\partial x_{i}}\right] w_{i}^{(0)} } & =-\frac{\partial Q_{i}}{\partial \sigma_{i}}, \quad x_{i}>B_{i}(t), t<T, \\
w_{i}^{(0)}\left(t, B_{i}(t)\right) & =0, \quad t \leq T, \\
w_{i}^{(0)}\left(T, x_{i}\right) & =0, \quad x_{i}>B_{i}(t), \\
{\left[\frac{\partial}{\partial t}+\frac{1}{2} \sigma_{i}^{2}(z) x_{i}^{2} \frac{\partial^{2}}{\partial x_{i}^{2}}+r x_{i} \frac{\partial}{\partial x_{i}}\right] w_{i}^{(1)} } & =-x_{i} \frac{\partial}{\partial x_{i}}\left(\frac{\partial Q_{i}}{\partial \sigma_{i}}\right), \quad x_{i}>B_{i}(t), t<T, \\
w_{i}^{(1)}\left(t, B_{i}(t)\right) & =0, \quad t \leq T, \\
w_{i}^{(1)}\left(T, x_{i}\right) & =0, \quad x_{i}>B_{i}(t),
\end{aligned}
$$




$$
\begin{aligned}
& {\left[\frac{\partial}{\partial t}+\frac{1}{2} \sigma_{i}^{2}(z) x_{i}^{2} \frac{\partial^{2}}{\partial x_{i}^{2}}+\frac{1}{2} \sigma_{j}^{2}(z) x_{j}^{2} \frac{\partial^{2}}{\partial x_{j}^{2}}+r x_{i} \frac{\partial}{\partial x_{i}}+r x_{j} \frac{\partial}{\partial x_{j}}\right] w_{i j}^{(1)}=-\left(x_{i} \frac{\partial Q_{i}}{\partial x_{i}}\right)\left(\frac{\partial Q_{j}}{\partial \sigma_{j}}\right),(29), \quad x_{i}>B_{i}(t), x_{j}>B_{j}(t), t<T,} \\
& w_{i j}^{(1)}\left(t, x_{i}, x_{j}\right)=0, \quad \text { if } \quad x_{i}=B_{i}(t) \quad \text { or } \quad x_{j}=B_{j}(t), t \leq T, \\
& w_{i j}^{(1)}\left(T, x_{i}, x_{j}\right)=0, \quad x_{i}>B_{i}(t), x_{j}>B_{j}(t) .
\end{aligned}
$$

Proof The proof is very similar to that of Proposition 2. Since $u_{0}$ depends on $z$ only through $\sigma_{i}(z)$ 's, we have

$$
\frac{\partial u_{0}}{\partial z}=\sum_{j=1}^{n} \sigma_{j}^{\prime}(z) \frac{\partial u_{0}}{\partial \sigma_{j}}
$$

and therefore

$$
\begin{aligned}
\sqrt{\delta}\left\langle\mathcal{M}_{1}\right\rangle u_{0} & =\nu_{Z} \sqrt{2 \delta}\left[\sum_{i=1}^{n} \rho_{i Z}\left\langle f_{i}(\cdot, z)\right\rangle x_{i} \frac{\partial}{\partial x_{i}}\left(\sum_{j=1}^{n} \sigma_{j}^{\prime}(z) \frac{\partial u_{0}}{\partial \sigma_{j}}\right)-\left\langle\Lambda_{2}(\cdot, z)\right\rangle \sum_{j=1}^{n} \sigma_{j}^{\prime}(z) \frac{\partial u_{0}}{\partial \sigma_{j}}\right] \\
& =\nu_{Z} \sqrt{2 \delta}\left[\sum_{i=1}^{n} \sum_{j=1}^{n} \rho_{i Z}\left\langle f_{i}(\cdot, z)\right\rangle \sigma_{j}^{\prime}(z) x_{i} \frac{\partial}{\partial x_{i}}\left(\frac{\partial u_{0}}{\partial \sigma_{j}}\right)-\left\langle\Lambda_{2}(\cdot, z)\right\rangle \sum_{i=1}^{n} \sigma_{i}^{\prime}(z) \frac{\partial u_{0}}{\partial \sigma_{i}}\right] \\
& =\sum_{i=1}^{n} R_{i}^{(0)} \frac{\partial u_{0}}{\partial \sigma_{i}}+\sum_{i=1}^{n} R_{i}^{(1)} x_{i} \frac{\partial}{\partial x_{i}}\left(\frac{\partial u_{0}}{\partial \sigma_{i}}\right)+\sum_{\substack{i, j=1 \\
i \neq j}}^{n} R_{i j}^{(1)} x_{i} \frac{\partial}{\partial x_{i}}\left(\frac{\partial u_{0}}{\partial \sigma_{j}}\right),
\end{aligned}
$$

where we have used the definitions $(24,25,26)$ of $\left(R_{i}^{(0)}, R_{i}^{(1)}, R_{i j}^{(1)}\right)$. By linearity of $(22)$, it is enough to check that

$$
\begin{aligned}
\left\langle\mathcal{L}_{2}\right\rangle\left(w_{i}^{(1)} \prod_{j=1, j \neq i}^{n} Q_{j}\right) & =-\frac{\partial u_{0}}{\partial \sigma_{i}} \\
\left\langle\mathcal{L}_{2}\right\rangle\left(w_{i}^{(1)} \prod_{j=1, j \neq i}^{n} Q_{j}\right) & =-x_{i} \frac{\partial}{\partial x_{i}}\left(\frac{\partial u_{0}}{\partial \sigma_{i}}\right), \\
\left\langle\mathcal{L}_{2}\right\rangle\left(w_{i j}^{(1)} \prod_{k=1, k \neq i, j}^{n} Q_{k}\right) & =-x_{i} \frac{\partial}{\partial x_{i}}\left(\frac{\partial u_{0}}{\partial \sigma_{j}}\right) .
\end{aligned}
$$

Using the form (7) of $\left\langle\mathcal{L}_{2}\right\rangle$ and $u_{0}=\prod_{i=1}^{n} Q_{i}$ one can easily check that (30), (31), and (32) are satisfied. The boundary and terminal conditions for the correction $\sqrt{\delta} u_{0,1}$ are directly inherited from the boundary and terminal conditions for the functions $w_{i}^{(0)}$ 's, $w_{i}^{(1)}$ 's, $w_{i j}^{(1)}$ 's, and $Q_{i}$ 's.

As for (16), (17) and (18) we show in Appendix B that the boundary value problems with sources (27), (28), and (29) for $w_{i}^{(0)}$ 's, $w_{i}^{(1)}$ 's, $w_{i j}^{(1)}$ 's can be transformed into boundary value problems without source leading to explicit formulas (up to Gaussian integrals). 


\subsection{Summary of the Approximation}

Combining the results of Propositions 1,2 and 3 we get that the approximation $\tilde{u}$ in (5) is given by

$$
\begin{aligned}
\tilde{u}=\prod_{i=1}^{n} Q_{i} & +\sum_{i=1}^{n} R_{i}^{(2)} w_{i}^{(2)} \prod_{\substack{j=1 \\
j \neq i}}^{n} Q_{j}+\sum_{i=1}^{n} R_{i}^{(3)} w_{i}^{(3)} \prod_{\substack{j=1 \\
j \neq i}}^{n} Q_{j}+\sum_{\substack{i, j=1 \\
i \neq j}}^{n} R_{i j}^{(3)} w_{i j}^{(3)} \prod_{\substack{k=1 \\
k \neq i, j}}^{n} Q_{k} \\
& +\sum_{i=1}^{n} R_{i}^{(0)} w_{i}^{(0)} \prod_{\substack{j=1 \\
j \neq i}}^{n} Q_{j}+\sum_{i=1}^{n} R_{i}^{(1)} w_{i}^{(1)} \prod_{\substack{j=1 \\
j \neq i}}^{n} Q_{j}+\sum_{\substack{i, j=1 \\
i \neq j}}^{n} R_{i j}^{(1)} w_{i j}^{(1)} \prod_{\substack{k=1 \\
k \neq i, j}}^{n} Q_{k},
\end{aligned}
$$

where $\left(R_{i}^{(2)}, R_{i}^{(3)}, R_{i j}^{(3)}\right)$ are small of order $\sqrt{\epsilon},\left(R_{i}^{(0)}, R_{i}^{(1)}, R_{i j}^{(1)}\right)$ are small of order $\sqrt{\delta}$, and they all depend on the parameter $z$. The functions $Q_{i}, w_{i}^{(2)}, w_{i}^{(3)}, w_{i}^{(0)}, w_{i}^{(1)}$ depend on the variable $x_{i}$, the functions $w_{i j}^{(3)}, w_{i j}^{(1)}$ depend on the variables $\left(x_{i}, x_{j}\right)$, and they all depend on the parameter $z$. The accuracy of approximation (33) is given at the end of Appendix A.

Remark The (approximated) survival probability of a single bond/firm (treated in Fouque et al. (2006)) is a particular case of the result (33). It can be obtained by fixing an index and eliminating all those terms that involve any one of the other indices, which includes all the twoindex cross-terms. For example, the (approximated) survival probability of bond 1 only involves $Q_{1}, w_{1}^{(0)}, w_{1}^{(1)}, w_{1}^{(2)}$ and $w_{1}^{(3)}$.

\subsection{Numerical Illustration of the Accuracy of Approximation}

In order to illustrate the quality of the approximation of the joint survival probability given by (33) we have conducted the following numerical experiments. For $n=10$ names (Table 1), and for $n=25$ names (Table 2), we compute the zero-order approximation $u_{0}$ given by (8), and the first order approximation $\tilde{u}$ given by (33) with the explicit formulas derived in Appendix B. We present the results for four sets of values of the small parameters $\epsilon$ and $\delta$. Since there is no explicit formulas for the true value, we obtain it by Monte Carlo simulations with a very large number of realizations $\left(10^{5}\right)$ and using an Euler scheme with a very small time-step $\left(10^{-4}\right)$ in order to ensure accuracy of the true value proxy denoted by $u_{\mathrm{MC}}$. The absolute and relative errors are shown in the last columns. In all cases we have used the following parameter values:

$$
\begin{aligned}
& X_{0}^{(i)}=20, r=5 \%, \eta_{i}=6 \%, K_{i}=10 \\
& \rho_{i j}=0, \rho_{i Y}=\rho_{i Z}=1 /(2 \sqrt{n}), \rho_{Y Z}=0 \\
& m_{Y}=m_{Z}=30 \%, \nu_{Y}=\nu_{Z}=10 \%, Y_{0}=Z_{0}=30 \%, \Lambda_{1}=\Lambda_{2}=0 \\
& f_{i}(y, z)=30 \% \exp (y+z) / \exp \left(m_{Y}+m_{Z}+\nu_{Y}^{2}+\nu_{Z}^{2}\right), T=1
\end{aligned}
$$

As expected the first order approximation $\tilde{u}$ converges to the (simulated) true value $u_{\mathrm{MC}}$ as $(\epsilon, \delta)$ goes to $(0,0)$. In fact, as often observed in homogenization, the approximation remains very 


\begin{tabular}{|c|c|c|c|c|c|}
\hline$\epsilon$ & $\delta$ & $u_{0}$ & $\tilde{u}$ & $u_{\mathrm{MC}}$ & Absolute (relative) error \\
\hline $1 / 100$ & $1 / 50$ & 0.740389 & 0.75079 & 0.7502 & $0.0006(0.08 \%)$ \\
$1 / 50$ & $1 / 20$ & 0.740389 & 0.756015 & 0.7529 & $0.003(0.4 \%)$ \\
$1 / 20$ & $1 / 10$ & 0.740389 & 0.763647 & 0.7567 & $0.007(0.9 \%)$ \\
1 & 1 & 0.740389 & 0.82833 & .7653 & $0.063(8.2 \%)$ \\
\hline
\end{tabular}

Table 1: Joint survival probability for ten firms $(n=10)$.

\begin{tabular}{|c|c|c|c|c|c|}
\hline$\epsilon$ & $\delta$ & $u_{0}$ & $\tilde{u}$ & $u_{\mathrm{MC}}$ & Absolute (relative) error \\
\hline $1 / 100$ & $1 / 50$ & 0.471683 & 0.481506 & 0.4789 & $0.003(0.5 \%)$ \\
$1 / 50$ & $1 / 20$ & 0.471683 & 0.486892 & 0.4803 & $0.006(1.4 \%)$ \\
$1 / 20$ & $1 / 20$ & 0.471683 & 0.488478 & 0.4854 & $0.003(0.6 \%)$ \\
$1 / 20$ & $1 / 10$ & 0.471683 & 0.493648 & 0.4843 & $0.009(1.9 \%)$ \\
\hline
\end{tabular}

Table 2: Joint survival probability for twenty five firms $(n=25)$.

accurate even in regimes where these parameters are not so small. We also observe that, in the present case with $\rho_{i j}=0$, the volatility-name correlations $\rho_{i Y}, \rho_{i Z}$ are small (since $\sum_{i=1}^{n} \rho_{i Y}^{2} \leq 1$ and $\left.\sum_{i=1}^{n} \rho_{i Z}^{2} \leq 1\right)$, and the effect of the correction due to stochastic volatility is also relatively small $\left(u_{0}\right.$ is already close to $\left.u_{\mathrm{MC}}\right)$. This will not be the case with name-name correlations $\rho_{i j} \neq 0$ as shown in Section 5.

\section{Loss Distribution}

We consider now a portfolio consisting of $N$ defaultable bonds and we denote by $a_{i}$ the number of bond $i$ in this portfolio. Assuming zero recovery rate from default for each bond, the loss at time $t$ of this portfolio is given by the random variable

$$
L(t) \equiv \sum_{i=1}^{N} a_{i} \chi_{i}(t),
$$

where $\chi_{i}(t)$ takes on 1 if bond $i$ defaults before $t$ and 0 otherwise. Our objective is to study the distribution of $L(T)$ for a maturity $T$ smaller than all the bond maturities.

\subsection{General Case}

Since the portfolio consists only of a finite number of bonds, $L$ must have a discrete distribution function. It hence suffices to find the probability value that a subset of names in the portfolio default. It turns out that this can be done recursively. For example,

$$
\begin{aligned}
& \mathbb{P}^{*}\{\text { only bond } 1 \text { defaults in the portfolio before time } T\} \\
& =\mathbb{P}^{*}\left\{\tau_{1} \leq T, \tau_{2}>T, \cdots, \tau_{N}>T\right\} \\
& =\mathbb{P}^{*}\left\{\tau_{2}>T, \cdots, \tau_{N}>T\right\}-\mathbb{P}^{*}\left\{\tau_{1}>T, \tau_{2}>T, \cdots, \tau_{N}>T\right\}
\end{aligned}
$$


while $\tau_{i}$ is the default time of bond $i$. Both terms on the right hand side of the last equality are known (approximately) by formula (33). In general, if we denote

$$
\begin{aligned}
& I \equiv\left\{i_{1}, i_{2}, \ldots, i_{n}\right\} \subset J \equiv\left\{j_{1}, j_{2}, \ldots, j_{m}\right\} \subset \bar{N} \equiv\{1,2, \ldots, N\}, \\
& \left.\left.D_{|J|}^{|I|}(I ; J) \equiv \mathbb{P}^{*}\{\text { (bond } i \text { defaults }, i \in I) \cap \text { (bond } j \text { survives, } j \in J \backslash I\right)\right\},
\end{aligned}
$$

then

$$
\begin{aligned}
D_{m}^{n}\left(\left\{i_{1}, \ldots, i_{n}\right\} ; J\right)= & \mathbb{P}^{*}\left\{\left(\tau_{i} \leq T, i \in\left\{i_{1}, \ldots, i_{n-1}, i_{n}\right\}\right) \cap\left(\tau_{j}>T, j \in J \backslash I\right)\right\} \\
= & \mathbb{P}^{*}\left\{\left(\tau_{i} \leq T, i \in\left\{i_{1}, \ldots, i_{n-1}\right\}\right) \cap\left(\tau_{j}>T, j \in J \backslash I\right)\right\} \\
& -\mathbb{P}^{*}\left\{\left(\tau_{i} \leq T, i \in\left\{i_{1}, \ldots, i_{n-1}\right\}\right) \cap\left(\tau_{j}>T, j \in J \backslash\left\{i_{1}, \ldots, i_{n-1}\right\}\right)\right\} .
\end{aligned}
$$

Therefore,

$$
\begin{aligned}
D_{m}^{n}\left(\left\{i_{1}, \ldots, i_{n}\right\} ;\left\{j_{1}, \ldots, j_{m}\right\}\right)= & D_{m-1}^{n-1}\left(\left\{i_{1}, \ldots, i_{n-1}\right\} ;\left\{j_{1}, \ldots, j_{m}\right\} \backslash\left\{i_{n}\right\}\right) \\
& -D_{m}^{n-1}\left(\left\{i_{1}, \ldots, i_{n-1}\right\} ;\left\{j_{1}, \ldots, j_{m}\right\}\right) .
\end{aligned}
$$

Formula (34) is recursive and by implementing it once, one can reduce by one the superscript $n$ (= the number of defaults) on $D$. This can be done repeatedly until one reduces the superscript to zero, implying a total survival of an appropriate subset of names in the portfolio. Finally the probability of total survival is (approximately) $\tilde{u}$ given by (33), with names/indices $\{1,2, \ldots, n\}$ replaced by the appropriate set of indices/names.

It can be shown that in order to compute $D_{m}^{n}(\cdot, \cdot)$, one has to evaluate $\tilde{u}$-like forms $2^{n}$ times. This may be computationally expensive for a portfolio of big size, say 50 names or more. For a smaller size portfolio, say $N=20$, however, the computational cost is acceptable to find for instance the probability of $50 \%$ loss, namely $D_{20}^{10}(\cdot, \cdot)$. To be more precise, the computation of $D_{20}^{10}(\cdot, \cdot)$ would require to compute $2^{10}=1024$ of $\tilde{u}$-like forms given by (33). However, every item in equation (33) is in closed-form (or up to double integrals), and therefore can be computed fairly fast when an appropriate programming language is chosen (say, $\mathrm{C} / \mathrm{C}++$ ). This can be done within 0.05 to 0.5 seconds on a $2 \mathrm{GHz}$ CPU with $2 \mathrm{~GB}$ RAM PC. Therefore the whole computation of $D_{20}^{10}(\cdot, \cdot)$ can be done within 51.2 to 512 seconds, which is what we mean by acceptable.

\subsection{Special Case: Homogeneous Portfolio}

We now consider a fully homogeneous portfolio, that is

$$
f_{i}(y, z)=f(y, z), \quad \rho_{i Y}=\rho_{Y}, \quad \rho_{i Z}=\rho_{Z}, \quad a_{i}=a, \quad X_{0}^{(i)}=x_{i}=x,
$$

for all $i=1, \cdots, N$. We first recall a classical result.

Lemma 1 Let $\tau_{i}$ be the default time of bond $i$, and suppose that under a probability $\mathbb{P}$

$$
\mathbb{P}\left(\tau_{i}>T, i \in\left\{i_{1}, i_{2}, \ldots, i_{m}\right\}\right)=\mathbb{P}\left(\tau_{j}>T, j \in\left\{j_{1}, j_{2}, \ldots, j_{m}\right\}\right),
$$

for any two equal-size sets of indices $\left\{i_{1}, i_{2}, \ldots, i_{m}\right\}$ and $\left\{j_{1}, j_{2}, \ldots, j_{m}\right\}$ chosen from $\{1,2, \ldots, N\}$ with $1 \leq m \leq N$. Define

$$
S_{m} \equiv \mathbb{P}\left(\tau_{i}>T, i \in\{1,2, \ldots, m\}\right), \quad 1 \leq m \leq N .
$$


Then, for $0 \leq k<N$, the probability that exactly $k$ bonds, among the $N$ bonds, default before $T$ is given by

$$
F_{k}^{(N)} \equiv \mathbb{P}\left(\sum_{i=1}^{N} \chi_{i}(T)=k\right)=\left(\begin{array}{l}
N \\
k
\end{array}\right) \sum_{j=0}^{k}\left(\begin{array}{l}
k \\
j
\end{array}\right)(-1)^{j} S_{N+j-k}
$$

Proof We provide here a short proof which consists in computing the moment generating function of the number of survivals. Denote by $\chi_{i}^{\prime} \equiv 1-\chi_{i}$ the event "firm $i$ survives after $T$ ".

On one hand we have

$$
\Phi(z) \equiv \mathbb{E}\left(z^{\left(\sum_{i=1}^{N} \chi_{i}^{\prime}\right)}\right)=\mathbb{E}\left(z^{\left(N-\sum_{i=1}^{N} \chi_{i}\right)}\right)=\sum_{k=0}^{N} z^{(N-k)} F_{k}^{(N)}=\sum_{k=0}^{N} z^{k} F_{N-k}^{(N)} .
$$

On the other hand we have

$$
\begin{aligned}
\Phi(z) & =\mathbb{E}\left(\prod_{i=1}^{N} z^{\chi_{i}^{\prime}}\right)=\mathbb{E}\left(\prod_{i=1}^{N}\left(1+(z-1) \chi_{i}^{\prime}\right)\right)=\mathbb{E}\left(\sum_{\left(i_{1}, \cdots, i_{n}\right), n=0, \cdots, N}(z-1)^{n} \chi_{i_{1}}^{\prime} \cdots \chi_{i_{n}}^{\prime}\right) \\
& =\sum_{n=0}^{N}(z-1)^{n} \sum_{\left(i_{1}, \cdots, i_{n}\right)} \mathbb{P}\left(\tau_{i}>T, i \in\left\{i_{1}, i_{2}, \ldots, i_{n}\right\}\right)=\sum_{n=0}^{N}(z-1)^{n}\left(\begin{array}{c}
N \\
n
\end{array}\right) S_{n} \\
& =\sum_{n=0}^{N}\left(\sum_{k=0}^{n}(-1)^{n-k}\left(\begin{array}{c}
n \\
k
\end{array}\right) z^{k}\right)\left(\begin{array}{c}
N \\
n
\end{array}\right) S_{n} \\
& =\sum_{k=0}^{N} z^{k}\left(\sum_{n=k}^{N}(-1)^{n-k}\left(\begin{array}{c}
n \\
k
\end{array}\right)\left(\begin{array}{c}
N \\
n
\end{array}\right) S_{n}\right) .
\end{aligned}
$$

The function $\Phi(z)$ being the polynomial in $z$ given by (38) and (39) we deduce that

$$
F_{N-k}^{(N)}=\sum_{n=k}^{N}(-1)^{n-k}\left(\begin{array}{c}
n \\
k
\end{array}\right)\left(\begin{array}{c}
N \\
n
\end{array}\right) S_{n}=\left(\begin{array}{c}
N \\
N-k
\end{array}\right) \sum_{n=k}^{N}(-1)^{n-k}\left(\begin{array}{c}
N-k \\
n-k
\end{array}\right) S_{n} .
$$

We finally obtain (37):

$$
\begin{aligned}
F_{k}^{(N)} & =\left(\begin{array}{c}
N \\
k
\end{array}\right) \sum_{n=N-k}^{N}(-1)^{n-N+k}\left(\begin{array}{c}
k \\
n-N+k
\end{array}\right) S_{n} \\
& =\left(\begin{array}{c}
N \\
k
\end{array}\right) \sum_{j=0}^{k}(-1)^{j}\left(\begin{array}{c}
k \\
j
\end{array}\right) S_{N-k+j},
\end{aligned}
$$

by setting $j=n-N+k$.

With the homogeneity assumption, we set $q \equiv Q_{1}(t, x)$ for the survival probability after $t$ of one given bond, and we obtain from the results in Section 3 that

$$
u_{0}=\prod_{i=1}^{n} Q_{i}=q^{n}
$$




$$
\begin{aligned}
\sqrt{\epsilon} u_{1,0} & =\sum_{i=1}^{n} R_{i}^{(2)} w_{i}^{(2)} \prod_{\substack{j=1 \\
j \neq i}}^{n} Q_{j}+\sum_{i=1}^{n} R_{i}^{(3)} w_{i}^{(3)} \prod_{\substack{j=1 \\
j \neq i}}^{n} Q_{j}+\sum_{\substack{i, j=1 \\
i \neq j}}^{n} R_{i j}^{(3)} w_{i j}^{(3)} \prod_{\substack{k=1 \\
k \neq i, j}}^{n} Q_{k} \\
& =n R_{1}^{(2)} w_{1}^{(2)}(t, x) q^{n-1}+n R_{1}^{(3)} w_{1}(t, x)^{(3)} q^{n-1}+n(n-1) R_{12}^{(3)} w_{12}^{(3)}(t, x, x) q^{n-2}, \\
\sqrt{\delta} u_{0,1} & =\sum_{i=1}^{n} R_{i}^{(0)} w_{i}^{(0)} \prod_{\substack{j=1 \\
j \neq i}}^{n} Q_{j}+\sum_{i=1}^{n} R_{i}^{(1)} w_{i}^{(1)} \prod_{\substack{j=1 \\
j \neq i}}^{n} Q_{j}+\sum_{\substack{i, j=1 \\
i \neq j}}^{n} R_{i j}^{(1)} w_{i j}^{(1)} \prod_{\substack{k=1 \\
k \neq i, j}}^{n} Q_{k} \\
& =n R_{1}^{(0)} w_{1}^{(0)}(t, x) q^{n-1}+n R_{1}^{(1)} w_{1}^{(1)}(t, x) q^{n-1}+n(n-1) R_{12}^{(1)} w_{12}^{(1)}(t, x, x) q^{n-2},
\end{aligned}
$$

where we have used the fact that the $R$ 's and w's do not depend on a particular choice of names $(i, j)$.

We define the quantities

$$
\begin{aligned}
A & \equiv \sum_{k=0}^{3} R_{1}^{(k)} w_{1}^{(k)}(t, x), \\
B & \equiv R_{12}^{(1)} w_{12}^{(1)}(t, x, x)+R_{12}^{(3)} w_{12}^{(3)}(t, x, x),
\end{aligned}
$$

which also depend on the parameter $z$, and we rewrite the joint survival probabilities (36) as

$$
S_{n} \approx \tilde{u} \equiv u_{0}+\sqrt{\epsilon} u_{1,0}+\sqrt{\delta} u_{0,1}=q^{n}+A n q^{n-1}+B n(n-1) q^{n-2}, \quad n \geq 2 .
$$

Note that $S_{1}=q+A$, and hence the previous formula for $S_{n}$ is actually valid for all $n \geq 1$, as well as for $n=0$ with $S_{0} \equiv 1$.

The approximated loss distribution is now given by

$$
\begin{aligned}
\mathbb{P}^{*}(L=k)= & \left(\begin{array}{l}
N \\
k
\end{array}\right) \sum_{j=0}^{k}\left(\begin{array}{c}
k \\
j
\end{array}\right)(-1)^{j} S_{N+j-k}=\left(\begin{array}{l}
N \\
k
\end{array}\right) \sum_{i=0}^{k}\left(\begin{array}{l}
k \\
i
\end{array}\right)(-1)^{k-i} S_{N-i} \\
\approx & \left(\begin{array}{c}
N \\
k
\end{array}\right) \sum_{i=0}^{k}\left(\begin{array}{c}
k \\
i
\end{array}\right)(-1)^{k-i}\left[q^{N-i}+A(N-i) q^{N-i-1}+B(N-i)(N-i-1) q^{N-i-2}\right] \\
= & \left(\begin{array}{c}
N \\
k
\end{array}\right) \sum_{i=0}^{k}\left(\begin{array}{c}
k \\
i
\end{array}\right)(-1)^{k-i} q^{N-i}+A\left(\begin{array}{c}
N \\
k
\end{array}\right) \sum_{i=0}^{k}\left(\begin{array}{c}
k \\
i
\end{array}\right)(-1)^{k-i}(N-i) q^{N-i-1} \\
& +B\left(\begin{array}{c}
N \\
k
\end{array}\right) \sum_{i=0}^{k}\left(\begin{array}{c}
k \\
i
\end{array}\right)(-1)^{k-i}(N-i)(N-i-1) q^{N-i-2} \\
\equiv & I_{0}+A I_{1}+B I_{2},
\end{aligned}
$$

where $I_{0}, I_{1}$, and $I_{2}$ are obtained by straightforward calculation:

$$
\begin{aligned}
I_{0} & =\left(\begin{array}{l}
N \\
k
\end{array}\right) \sum_{i=0}^{k}\left(\begin{array}{c}
k \\
i
\end{array}\right)(-1)^{k-i} q^{N-i}=\left(\begin{array}{c}
N \\
k
\end{array}\right)(1-q)^{k} q^{N-k}, \\
I_{1} & =\left(\begin{array}{c}
N \\
k
\end{array}\right)\left[\sum_{i=0}^{k}\left(\begin{array}{c}
k \\
i
\end{array}\right)(-1)^{k-i} s^{N-i}\right]_{s=q}^{\prime}=\left(\begin{array}{c}
N \\
k
\end{array}\right)\left[s^{N-k}(1-s)^{k}\right]_{s=q}^{\prime} \\
& =\left(\begin{array}{c}
N \\
k
\end{array}\right)\left[(N-k) q^{N-k-1}(1-q)^{k}-k q^{N-k}(1-q)^{k-1}\right]
\end{aligned}
$$




$$
\begin{aligned}
= & {\left[\frac{N-k}{q}-\frac{k}{1-q}\right] I_{0}, } \\
I_{2}= & \left(\begin{array}{l}
N \\
k
\end{array}\right)\left[\sum_{i=0}^{k}\left(\begin{array}{c}
k \\
i
\end{array}\right)(-1)^{k-i} s^{N-i}\right]_{s=q}^{\prime \prime}=\left(\begin{array}{c}
N \\
k
\end{array}\right)\left[s^{N-k}(1-s)^{k}\right]_{s=q}^{\prime \prime} \\
= & \left(\begin{array}{c}
N \\
k
\end{array}\right)\left[(N-k)(N-k-1) q^{N-k-2}(1-q)^{k}-2 k(N-k) q^{N-k-1}(1-q)^{k-1}\right. \\
& \left.+k(k-1) q^{N-k}(1-q)^{k-2}\right] \\
= & {\left[\frac{(N-k)(N-k-1)}{q^{2}}-\frac{2 k(N-k)}{q(1-q)}+\frac{k(k-1)}{(1-q)^{2}}\right] I_{0} . }
\end{aligned}
$$

To summarize, for $0 \leq k<N$,

$$
\mathbb{P}^{*}(L=k) \approx I_{0}+A I_{1}+B I_{2}
$$

where $I_{0}, I_{1}$ and $I_{2}$ are explicitly given by $(42,43,44)$, and $A$ and $B$ defined in $(40,41)$ are small of order $\max \{\sqrt{\epsilon}, \sqrt{\delta}\}$.

Note that $I_{0}$ corresponds to the case where all the underlying assets are mutually independent, which gives rise to the classic binomial distribution. Since the method used in this paper is perturbation, we call (45) the perturbed binomial formula.

\subsection{Numerical Illustration}

In the homogeneous-portfolio case discussed above, we implemented some numerical computation to illustrate the effect of introducing stochastic volatilities. We compute the approximated loss distribution given by (45) where only four parameters $(N, q, A, B)$ are needed. The purpose of this computation is to show stylized features of the loss distribution generated by the correlation of defaults due to the presence of stochastic volatility.

The parameters used in Figure 1 are as follows:

$$
N=100, \quad q=0.9, \quad A=0.00, \quad B=0.0006 .
$$

The upper graph presents the probability mass function and the lower one presents the cumulative distribution function for the loss. Note that in order to make comparison between our result and the classic binomial distribution, we chose $A=0$ because a non-zero $A$ would make single name survival probabilities distinct under these two scenarios. By choosing $A=0$, the single name survival probability is $q=0.9=90 \%$. Note also that $B$ is chosen very small to ensure that the approximation is in its range of validity.

It can be observed from Figure 1 that:

- the probability, with stochastic volatilities, that the total portfolio loss is less than $3 \%$ is bigger than that from the classic binomial distribution; 

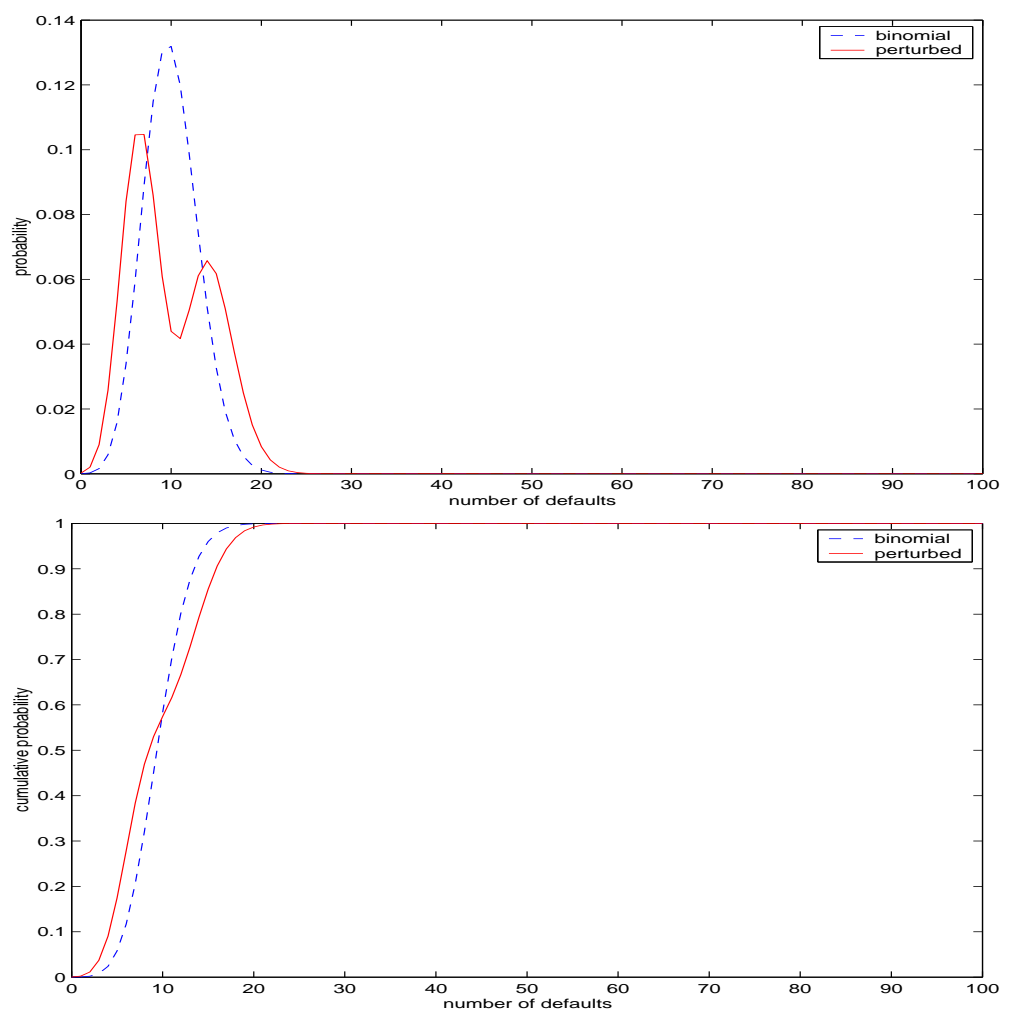

Figure 1: Perturbed Binomial Loss Distribution

- the probability, with stochastic volatilities, that the total portfolio loss is bigger than $15 \%$ is bigger than that from the classic binomial distribution.

In terms of CDO tranches (we refer to Duffie and Singleton (2003) for an introduction), this implies that the expected loss and hence the fair spread of the equity tranche become less, while the expected loss and hence the fair spread of the senior tranche become bigger, than in the classic binomial case. This phenomenon is consistent with what has been documented so far in CDO literature if one increases the default correlation between the underlying bonds and keeps other things unchanged. For example, see Duffie and Garleanu (2001). Note that a positive number $B$ indicates positive correlation between the underlying bonds. Here we assume that the equity tranche absorbs 0-3\% loss and the senior absorbs 15\%-100\% loss.

Furthermore, the binomial probability mass function achieves its maximum 0.1319 at $k=11$, while the probability mass function with stochastic volatilities achieves its maximum 0.1047 at $k=8$.

\section{Models with Name-Name Correlation}

We now consider the correlated case, namely where the Brownian motions $\tilde{W}^{(i)}$ 's driving the names are correlated. Using the notation of Section 2, this means taking $\mathrm{d}\left\langle\tilde{W}^{(i)}, \tilde{W}^{(j)}\right\rangle_{t}=\rho_{i j} \mathrm{~d} t$ for $i \neq j$ 
under the risk-neutral probability measure $\tilde{\mathbb{P}}$ with $\left|\rho_{i j}\right|<1$. Observe that for non-zero $\rho_{i j}$ 's the conditions $\sum_{i=1}^{n} \rho_{i Y}^{2} \leq 1$ and $\sum_{i=1}^{n} \rho_{i Z}^{2} \leq 1$ are no longer needed. We denote by $u^{\epsilon, \delta, \rho}$ the joint survival probability defined by

$$
u^{\epsilon, \delta, \rho}(t, \mathbf{x}, y, z) \equiv \tilde{\mathbb{P}}\left\{\tau_{t}^{(1)}>T, \ldots, \tau_{t}^{(n)}>T \mid \mathbf{X}_{t}=\mathbf{x}, Y_{t}=y, Z_{t}=z\right\}
$$

In terms of PDEs, $u^{\epsilon, \delta, \rho}$ is the solution to the boundary value problem

$$
\begin{aligned}
\mathcal{L}^{\epsilon, \delta, \rho} u^{\epsilon, \delta, \rho} & =0, \quad x_{i}>B_{i}(t), \text { for all } i, t<T, \\
u^{\epsilon, \delta, \rho}\left(t, x_{1}, x_{2}, \ldots, x_{n}, y, z\right) & =0, \quad \exists i \in\{1, \cdots, n\}, x_{i}=B_{i}(t), t \leq T, \\
u^{\epsilon, \delta, \rho}\left(T, x_{1}, x_{2}, \ldots, x_{n}, y, z\right) & =1, \quad x_{i}>B_{i}(t), \text { for all } i,
\end{aligned}
$$

where the operator $\mathcal{L}^{\epsilon, \delta, \rho}$ can be written as

$$
\mathcal{L}^{\epsilon, \delta, \rho}=\mathcal{L}^{\epsilon, \delta}+\sum_{i<j}^{n} \rho_{i j} \mathcal{L}_{\rho}^{(i j)},
$$

where we have used $\rho_{i j}=\rho_{j i}$, the definition (2) of the operator $\mathcal{L}^{\epsilon, \delta}$, and the notation

$$
\mathcal{L}_{\rho}^{(i j)}=f_{i}(y, z) f_{j}(y, z) x_{i} x_{j} \frac{\partial^{2}}{\partial x_{i} \partial x_{j}} .
$$

The leading order term in the small $\epsilon$ and small $\delta$ expansion carried out in the previous sections would correspond to the correlated multi-name case with constant volatility. As explained in the introduction this is not a tractable model because of the lack of simple formulas for the joint distribution of hitting times. This leads us to perform an additional expansion around the independent case where the $\rho_{i j}$ 's are zero. We therefore consider the case where the $\rho_{i j}$ 's are small and of the same order.

Expanding $u^{\epsilon, \delta, \rho}$ in powers of $\sqrt{\epsilon}, \sqrt{\delta}$, and $\rho_{i j}$ we get:

$$
u^{\epsilon, \delta, \rho}=u^{\epsilon, \delta}+\sum_{i<j}^{n} \rho_{i j}\left(u_{0,0,1}^{(i j)}+\sqrt{\epsilon} u_{1,0,1}^{(i j)}+\sqrt{\delta} u_{0,1,1}^{(i j)}+\cdots\right)+\cdots,
$$

and retain the lowest order terms,

$$
\tilde{u} \equiv u_{0}+\sqrt{\epsilon} u_{1,0}+\sqrt{\delta} u_{0,1}+\sum_{i<j}^{n} \rho_{i j} u_{0,0,1}^{(i j)},
$$

as our approximation for $u^{\epsilon, \delta, \rho}$ where the first three terms have been computed in Section 3 .

\subsection{Correction Terms $\rho_{i j} u_{0,0,1}^{(i j)}$}

From Appendix A the term $u_{0,0,1}^{(i j)}$ is determined by the following PDE system:

$$
\begin{aligned}
\left\langle\mathcal{L}_{2}\right\rangle u_{0,0,1}^{(i j)} & =-\left\langle\mathcal{L}_{\rho}^{(i j)}\right\rangle u_{0}, \quad x_{l}>B_{l}(t), \text { for all } l, t<T, \\
u_{0,0,1}^{(i j)}\left(t, x_{1}, x_{2}, \ldots, x_{n}\right) & =0, \quad \exists l \in\{1, \cdots, n\}, x_{l}=B_{l}(t), t \leq T, \\
u_{0,0,1}^{(i j)}\left(T, x_{1}, x_{2}, \ldots, x_{n}\right) & =0, \quad x_{l}>B_{l}(t), \text { for all } l,
\end{aligned}
$$


where the operator $\left\langle\mathcal{L}_{\rho}^{(i j)}\right\rangle$ is given by

$$
\left\langle\mathcal{L}_{\rho}^{(i j)}\right\rangle=\left\langle f_{i}(\cdot, z) f_{j}(\cdot, z)\right\rangle x_{i} x_{j} \frac{\partial^{2}}{\partial x_{i} \partial x_{j}} .
$$

The following result shows that each $u_{0,0,1}^{(i j)}$ corresponds to a single two-dimensional problem.

Proposition 4 The correction term $\rho_{i j} u_{0,0,1}^{(i j)}$ is given by

$$
\rho_{i j} u_{0,0,1}^{(i j)}=R_{i j}^{(4)} w_{i j}^{(4)} \prod_{\substack{k=1 \\ k \neq i, j}}^{n} Q_{k},
$$

where the coefficient $R_{i j}^{(4)}$ depends on the parameter $z$ and is given by

$$
R_{i j}^{(4)}=\rho_{i j}\left\langle f_{i}(\cdot, z) f_{j}(\cdot, z)\right\rangle, i \neq j,
$$

and where the function $w_{i j}^{(4)}\left(t, x_{i}, x_{j}\right)$ depends on the parameter $z$ and is given by the following problem:

$$
\begin{aligned}
& {\left[\frac{\partial}{\partial t}+\frac{1}{2} \sigma_{i}^{2}(z) x_{i}^{2} \frac{\partial^{2}}{\partial x_{i}^{2}}+\frac{1}{2} \sigma_{j}^{2}(z) x_{j}^{2} \frac{\partial^{2}}{\partial x_{j}^{2}}+r x_{i} \frac{\partial}{\partial x_{i}}+r x_{j} \frac{\partial}{\partial x_{j}}\right] w_{i j}^{(4)}=-\left(x_{i} \frac{\partial Q_{i}}{\partial x_{i}}\right)\left(x_{j} \frac{\partial Q_{j}}{\partial x_{j}}\right)(, 54)} \\
& w_{i j}^{(4)}\left(t, x_{i}, x_{j}\right)=0, \quad \text { if } \quad x_{i}=B_{i}(t), x_{j}>B_{j}(t), t<T, \\
& w_{i j}^{(4)}\left(T, x_{i}, x_{j}\right)=0, \quad x_{i}>B_{i}(t), x_{j}>B_{j}(t),
\end{aligned}
$$

with $\sigma_{i}(z)$ given in Proposition 1.

Proof The proof is very similar to that of Proposition 2. With the definition (53) of $R_{i j}^{(4)}$, we have

$$
\rho_{i j}\left\langle\mathcal{L}_{\rho}^{(i j)}\right\rangle u_{0}=R_{i j}^{(4)} x_{i} \frac{\partial}{\partial x_{i}}\left(x_{j} \frac{\partial u_{0}}{\partial x_{j}}\right) .
$$

It is therefore enough to check that

$$
\left\langle\mathcal{L}_{2}\right\rangle\left(w_{i j}^{(4)} \prod_{k=1, k \neq i, j}^{n} Q_{k}\right)=-x_{i} \frac{\partial}{\partial x_{i}}\left(x_{j} \frac{\partial u_{0}}{\partial x_{j}}\right) .
$$

Using the form (7) of $\left\langle\mathcal{L}_{2}\right\rangle$ and $u_{0}=\prod_{i=1}^{n} Q_{i}$ one can easily check that (56) is satisfied. The boundary and terminal conditions for the correction $\rho_{i j} u_{0,0,1}^{(i j)}$ are directly inherited from the boundary and terminal conditions for the functions $w_{i j}^{(4)}$ 's, and $Q_{i}$ 's.

As for (18) we show in Appendix B that the boundary value problem with source (54) for $w_{i j}^{(4)}$ can be transformed into boundary value problems without source leading to explicit formulas (up to Gaussian integrals). 


\subsection{Summary of Approximation with Name-Name Correlation}

Combining the results of Propositions (1)-(4), we get that the approximation $\tilde{u}$ in (50) is given by

$$
\begin{aligned}
\tilde{u}=\prod_{i=1}^{n} Q_{i} & +\sum_{i=1}^{n} R_{i}^{(2)} w_{i}^{(2)} \prod_{\substack{j=1 \\
j \neq i}}^{n} Q_{j}+\sum_{i=1}^{n} R_{i}^{(3)} w_{i}^{(3)} \prod_{\substack{j=1 \\
j \neq i}}^{n} Q_{j}+\sum_{\substack{i, j=1 \\
i \neq j}}^{n} R_{i j}^{(3)} w_{i j}^{(3)} \prod_{\substack{k=1 \\
k \neq i, j}}^{n} Q_{k} \\
& +\sum_{i=1}^{n} R_{i}^{(0)} w_{i}^{(0)} \prod_{\substack{j=1 \\
j \neq i}}^{n} Q_{j}+\sum_{i=1}^{n} R_{i}^{(1)} w_{i}^{(1)} \prod_{\substack{j=1 \\
j \neq i}}^{n} Q_{j}+\sum_{\substack{i, j=1 \\
i \neq j}}^{n} R_{i j}^{(1)} w_{i j}^{(1)} \prod_{\substack{k=1 \\
k \neq i, j}}^{n} Q_{k} \\
& +\sum_{i<j}^{n} R_{i j}^{(4)} w_{i j}^{(4)} \prod_{\substack{k=1 \\
k \neq i, j}}^{n} Q_{k},
\end{aligned}
$$

where $R_{i j}^{(4)}$ are small of order $\rho_{i j}$ and depend on the parameter $z$, and the functions $w_{i j}^{(4)}$ depend on the variables $\left(x_{i}, x_{j}\right)$ and the parameter $z$.

\subsection{Loss Distribution (Homogeneous Portfolio)}

For simplicity we only consider the homogeneous portfolio case. In addition to the homogeneity conditions (35) we also assume that $\rho_{i j}=\rho$ for all $(i, j)$ and $|\rho| \ll 1$.

Using the same notation as in Section 4, the name-name correction term becomes:

$$
\begin{aligned}
\sum_{i<j}^{n} \rho_{i j} u_{0,0,1}^{(i j)} & =\sum_{i<j}^{n} R_{i j}^{(4)} w_{i j}^{(4)} \prod_{\substack{k=1 \\
k \neq i, j}}^{n} Q_{k} \\
& =\frac{1}{2} n(n-1) R_{12}^{(4)} w_{12}^{(4)}(t, x, x) q^{n-2}
\end{aligned}
$$

where from (53), $R_{12}^{(4)}=\rho \sigma^{2}(z)$.

In particular this implies that the approximation for the loss distribution given by (45) still holds if we replace the quantity $B$ in (41) by $B+B_{\rho}$ where

$$
B_{\rho} \equiv \frac{1}{2} R_{12}^{(4)} w_{12}^{(4)}(t, x, x),
$$

so that (45) becomes

$$
\mathbb{P}^{*}(L=k) \approx I_{0}+A I_{1}+\left(B+B_{\rho}\right) I_{2}
$$

Note that for a single maturity $T$ the correlations generated by stochastic volatility and namename correlation are of the same form to leading order. However if one looks at the term structure of correlation across several maturities, an important aspect of CDO tranches, then the shape of the function $w_{12}^{(4)}$ is different from the shapes of $w_{12}^{(1)}$ and $w_{12}^{(3)}$ and therefore the nature of the correlation plays a role. 


\subsection{Numerical Illustration}

In order to illustrate the quality of the approximation of the joint survival probability given by $(57)$ we have conducted the following numerical experiments. For $n=25$ names (Table 3), we compute the zero-order approximation $u_{0}$ given by (8), and the first order approximation $\tilde{u}$ given by $(57)$ and the explicit formulas derived in Appendix B. We present the results for $\epsilon=0.02, \delta=0.5$, and five cases of $\rho_{i j}=\rho$. The true value proxy $u_{\mathrm{MC}}$ is obtained by Monte Carlo simulations with $10^{5}$ realizations and using an Euler scheme with time-step of $10^{-4}$ as in Section 3.6. The other parameters are as in Section 3.6.

\begin{tabular}{|c|c|c|c|c|c|c|}
\hline$\epsilon$ & $\delta$ & $\rho_{i j}$ & $u_{0}$ & $\tilde{u}$ & $u_{\mathrm{MC}}$ & Absolute (relative) error \\
\hline $1 / 50$ & $1 / 20$ & 0 & 0.471683 & 0.486892 & 0.4803 & $0.006(1.4 \%)$ \\
$1 / 50$ & $1 / 20$ & 0.05 & 0.471683 & 0.518151 & 0.5119 & $0.006(1.2 \%)$ \\
$1 / 50$ & $1 / 20$ & 0.1 & 0.471683 & 0.549409 & 0.5426 & $0.007(1.3 \%)$ \\
$1 / 50$ & $1 / 20$ & 0.2 & 0.471683 & 0.611926 & 0.5986 & $0.013(2.2 \%)$ \\
$1 / 50$ & $1 / 20$ & 0.4 & 0.471683 & 0.736961 & 0.6937 & $0.043(6.2 \%)$ \\
\hline
\end{tabular}

Table 3: Joint survival probability for twenty five firms $(n=25)$ with stochastic volatility and name-name correlations.

The presence of a small name-name correlation $\rho$ enhances the importance of the correction $\left(\tilde{u}-u_{0}\right)$ derived in this paper, as can be seen by comparing $u_{0}$ and $u_{\mathrm{MC}}$ in Table 2 and in Table 3.

Table 4 gives a loss distribution generated with stochastic volatility and name-name correlation, as computed by (59). The results are plotted in Figure 2. The parameters are taken from Section 3.6, with $n=100, \epsilon=1 / 50, \delta=1 / 20, \rho_{i j}=1 / 10$. With these parameters, the coefficients in (59) are: $A=6.607 \times 10^{-4}, B=-0.014 \times 10^{-4}$, and $B_{\rho}=2.08 \times 10^{-4}$. The binomial distribution plotted in Figure 2 is obtained by setting $A=B=B_{\rho}=0$ in (59).

\begin{tabular}{|c|c|c|c|c|}
\hline 0 & 1 & 2 & 3 & 4 \\
0.16 & 0.26 & 0.17 & 0.062 & 0.047 \\
\hline \hline 5 & 6 & 7 & 8 & 9 \\
0.078 & 0.086 & 0.065 & 0.037 & 0.017 \\
\hline \hline 10 & 11 & 12 & 13 & 14 \\
$6.5 \times 10^{-3}$ & $2.2 \times 10^{-3}$ & $6.2 \times 10^{-4}$ & $1.6 \times 10^{-4}$ & $3.7 \times 10^{-5}$ \\
\hline
\end{tabular}

Table 4: Loss distribution for 100 firms $(n=100)$ with stochastic volatility and name-name correlations. All omitted probabilities are less than $10^{-5}$. The expected number of defaults is 2.88 when stochastic volatility is present, while it is 2.96 in the independent constant volatility case.

The loss distribution shown in Figure 2 clearly has a bimodal structure. With the given parameters, one can see that the event of having $2-5$ defaults is significantly less likely than if the stocks were independent. 


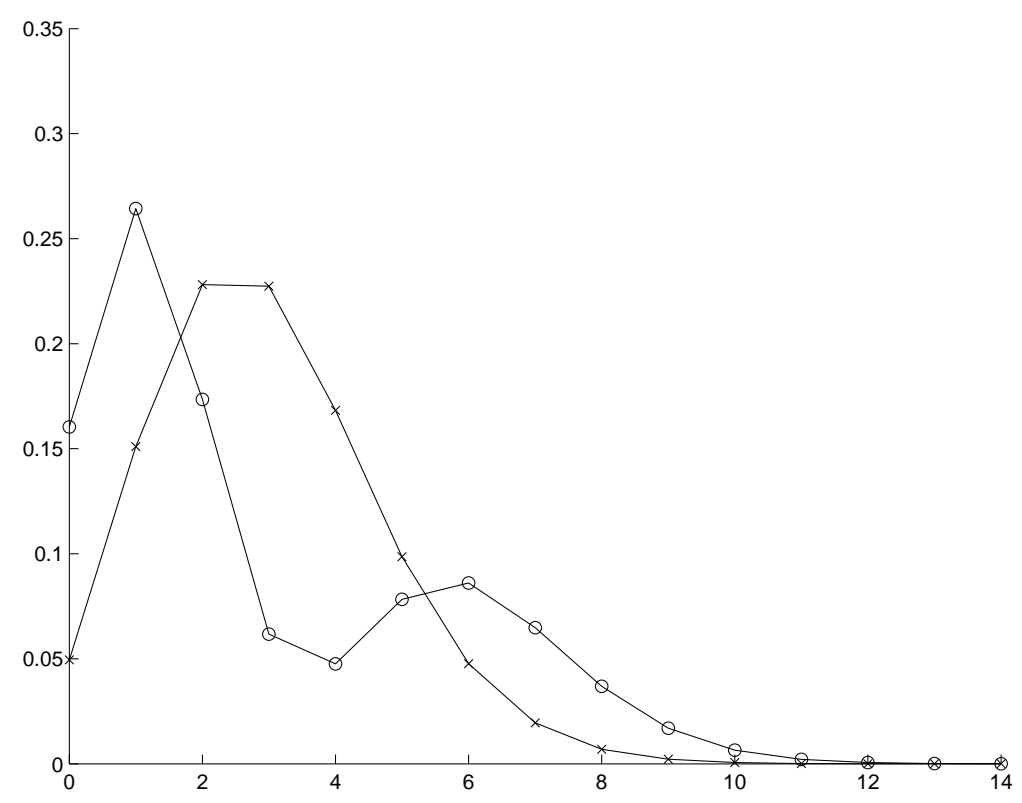

Figure 2: Plot of the loss distribution (o) given in Table 4. The mass function of the corresponding binomial distribution $(\times)$ is superimposed for comparison.

\section{A Formal Expansion}

Following Fouque et al. (2003), we present the formal expansion used in Section 3, and we discuss its accuracy. We compute $\mathcal{L}^{\epsilon, \delta} u^{\epsilon, \delta}$ where the operator $\mathcal{L}^{\epsilon, \delta}$ is given by (2), and the function $u^{\epsilon, \delta}$ is first expanded in powers of $\sqrt{\delta}$ corresponding to a regular perturbation:

$$
u^{\epsilon, \delta}=u_{0}^{\epsilon}+\sqrt{\delta} u_{1}^{\epsilon}+\delta u_{2}^{\epsilon}+\cdots
$$

In order to cancel the terms of order zero in $\delta$ and the terms of order $\sqrt{\delta}$ in $\mathcal{L}^{\epsilon, \delta} u^{\epsilon, \delta}=0$, we get

$$
\begin{aligned}
\left(\frac{1}{\epsilon} \mathcal{L}_{0}+\frac{1}{\sqrt{\epsilon}} \mathcal{L}_{1}+\mathcal{L}_{2}\right) u_{0}^{\epsilon} & =0 \\
\left(\frac{1}{\epsilon} \mathcal{L}_{0}+\frac{1}{\sqrt{\epsilon}} \mathcal{L}_{1}+\mathcal{L}_{2}\right) u_{1}^{\epsilon}+\mathcal{M}_{1} u_{0}^{\epsilon}+\frac{1}{\sqrt{\epsilon}} \mathcal{M}_{3} u_{0}^{\epsilon} & =0,
\end{aligned}
$$

with zero boundary value conditions at $x_{i}=B_{i}(t)$ and a terminal condition one (resp. zero) at $t=T$ for $u_{0}^{\epsilon}$ (resp. $u_{1}^{\epsilon}$ ). We now expand $u_{0}^{\epsilon}$ in powers of $\sqrt{\epsilon}$ corresponding to a singular perturbation:

$$
u^{\epsilon}=u_{0}+\sqrt{\epsilon} u_{1,0}+\epsilon u_{2,0}+\epsilon \sqrt{\epsilon} u_{3,0} \cdots
$$

so that (61) becomes:

$$
\begin{aligned}
\frac{1}{\epsilon} \mathcal{L}_{0} u_{0} & +\frac{1}{\sqrt{\epsilon}}\left(\mathcal{L}_{0} u_{1,0}+\mathcal{L}_{1} u_{0}\right)+\left(\mathcal{L}_{0} u_{2,0}+\mathcal{L}_{1} u_{1,0}+\mathcal{L}_{2} u_{0}\right) \\
& +\sqrt{\epsilon}\left(\mathcal{L}_{0} u_{3,0}+\mathcal{L}_{1} u_{2,0}+\mathcal{L}_{2} u_{1,0}\right)+\cdots=0
\end{aligned}
$$

By choosing $u_{0}$ and $u_{1,0}$ independent of the variable $y$ the terms in $1 / \epsilon$ and $1 / \sqrt{\epsilon}$ cancel. The operator $\mathcal{L}_{1}$ taking derivatives with respect to $y$, the next term becomes

$$
\mathcal{L}_{0} u_{2,0}+\mathcal{L}_{2} u_{0}=0
$$


which is a Poisson equation in $u_{2,0}$ with respect to the variable $y$, leading to the solvability condition

$$
\left\langle\mathcal{L}_{2}\right\rangle u_{0}=0,
$$

and the problem (6) characterizing $u_{0}$. Subsequently $u_{2,0}$ is given by $-\mathcal{L}_{0}^{-1}\left(\mathcal{L}_{2}-\left\langle\mathcal{L}_{2}\right\rangle\right) u_{0}$, and the terms of order $\sqrt{\epsilon}$, as a Poisson equation in $u_{3,0}$ with respect to the variable $y$, lead to the solvability condition

$$
\left\langle\mathcal{L}_{2}\right\rangle u_{1,0}=\left\langle\mathcal{L}_{1} \mathcal{L}_{0}^{-1}\left(\mathcal{L}_{2}-\left\langle\mathcal{L}_{2}\right\rangle\right)\right\rangle u_{0}
$$

and the problem (9) characterizing $u_{1,0}$.

In order to obtain $u_{0,1}$ needed in the approximation (5), we only need the leading order term in $u_{1}^{\epsilon}=u_{0,1}+\sqrt{\epsilon} u_{1,1}+\cdots$ since $u_{1}^{\epsilon}$ is multiplied by $\sqrt{\delta}$ in (60). By choosing $u_{0,1}$ and $u_{1,1}$ independent of the variable $y$, and using the fact that $\mathcal{M}_{3}$ takes derivatives with respect to $y$, to leading order equation (62) becomes:

$$
\mathcal{L}_{0} u_{2,1}+\mathcal{L}_{2} u_{0,1}+\mathcal{M}_{1} u_{0}=0
$$

which is again a Poisson equation in $u_{2,1}$ with respect to $y$. Its solvability condition

$$
\left\langle\mathcal{L}_{2}\right\rangle u_{0,1}=-\left\langle\mathcal{M}_{1}\right\rangle u_{0}
$$

leads to the problem (22) characterizing $u_{0,1}$.

The accuracy of the the approximation (5) is the same as obtained in Fouque et al. (2006), that is $\mathcal{O}\left(\epsilon^{2 / 3} \log |\epsilon|+\delta\right)$.

The computation of $\mathcal{L}^{\epsilon, \delta, \rho} u^{\epsilon, \delta, \rho}$, used in Section 5, proceeds along the same lines. The operator $\mathcal{L}^{\epsilon, \delta, \rho}$ is given by (47), and the function $u^{\epsilon, \delta, \rho}$ is first expanded in powers of $\rho_{i j}$ corresponding to a regular perturbation:

$$
u^{\epsilon, \delta, \rho}=u_{0}^{\epsilon, \delta}+\sum_{i<j}^{n} \rho_{i j} u_{1}^{\epsilon, \delta,(i j)}+\sum_{i<j}^{n} \sum_{k<l}^{n} \rho_{i j} \rho_{k l} u_{2}^{\epsilon, \delta,(i j ; k l)}+\cdots
$$

Canceling the terms of order zero in $\rho_{i j}$ and the terms of order $\rho_{i j}$ in $\mathcal{L}^{\epsilon, \delta, \rho} u^{\epsilon, \delta, \rho}=0$, we get

$$
\begin{aligned}
& \mathcal{L}^{\epsilon, \delta} u_{0}^{\epsilon, \delta}=0 \\
& \mathcal{L}^{\epsilon, \delta} u_{1}^{\epsilon, \delta}=-\mathcal{L}_{\rho}^{(i j)} u_{0}^{\epsilon, \delta},
\end{aligned}
$$

with zero boundary conditions at $x_{i}=B_{i}(t)$ and a terminal condition one (resp. zero) at $t=T$ for $u_{0}^{\epsilon, \delta}$ (resp. $u_{1}^{\epsilon, \delta}$ ) where $\mathcal{L}_{\rho}^{(i j)}$ is given by (48). Equation (64) has been discussed above. Equation (65) has a source which is of order zero in $\epsilon$ and in $\delta$, so that after expanding first in $\sqrt{\delta}$ and then in $\sqrt{\epsilon}, u_{0,0,1}^{(i j)}$ and $u_{1,0,1}^{(i j)}$ can be chosen independent of $y$ to make the terms in $1 / \epsilon$ and $1 / \sqrt{\epsilon}$ cancel. After dropping terms which differentiate $u_{0,0,1}^{(i j)}$ or $u_{1,0,1}^{(i j)}$ with respect to $y$, to leading order equation (65) becomes

$$
\mathcal{L}_{0} u_{2,0,1}^{(i j)}+\mathcal{L}_{2} u_{0,0,1}^{(i j)}=-\mathcal{L}_{\rho}^{(i j)} u_{0,0,0} .
$$

This Poisson equation has the solvability condition

$$
\left\langle\mathcal{L}_{2}\right\rangle u_{0,0,1}^{(i j)}=-\left\langle\mathcal{L}_{\rho}^{(i j)}\right\rangle u_{0,0,0}
$$

which is the problem (51) characterizing $u_{0,0,1}^{(i j)}$. 


\section{B Explicit Formulas}

In order to implement the main formulas (33) and (58) for the approximation $\tilde{u}$, one needs to compute the functions $w_{i}^{(l)}$ for each $i \in\{1,2, \cdots, n\}, l \in\{0,1,2,3\}$, and $w_{i j}^{(l)}$ for each pair $(i, j) \in$ $\{1,2, \cdots, n\} \times\{1,2, \cdots, n\}$ with $i \neq j$, and $l \in\{1,3,4\}$, defined in $(16,17,18,27,28,29,54)$.

We present in this Appendix an efficient method for computing these quantities. The formulas are given below in Lemmas 2 and 3 .

Lemma 2 For each $i \in\{1,2, \cdots, n\}$ :

a) $w_{i}^{(2)}$ is given by

$$
w_{i}^{(2)}\left(t, x_{i}\right)=\hat{w}_{i}^{(2)}\left(t, x_{i}\right)-(T-t) F_{i}^{(2)}\left(t, x_{i}\right)
$$

where

$$
\begin{aligned}
& F_{i}^{(2)}\left(t, x_{i}\right) \equiv x_{i}^{2} \frac{\partial^{2} Q_{i}}{\partial x_{i}^{2}} \\
& =\mathrm{N}^{\prime}\left(d_{2(i)}^{+}\right)\left[-\frac{d_{2(i)}^{+}}{\sigma_{i}^{2}(T-t)}-\frac{1}{\sigma_{i} \sqrt{T-t}}\right]-\mathrm{N}\left(d_{2(i)}^{-}\right) p_{i}\left(p_{i}-1\right)\left(\frac{x_{i}}{B_{i}(t)}\right)^{p_{i}} \\
& +\mathrm{N}^{\prime}\left(d_{2(i)}^{-}\right)\left[\frac{d_{2(i)}^{-}}{\sigma_{i}^{2}(T-t)}+\frac{2 p_{i}-1}{\sigma_{i} \sqrt{T-t}}\right]\left(\frac{x_{i}}{B_{i}(t)}\right)^{p_{i}}, \\
& \hat{w}_{i}^{(2)}\left(t, x_{i}\right)=\int_{t}^{T} g_{i}^{(2)}(s) h_{i}\left(s, x_{i}\right) \mathrm{d} s, \\
& g_{i}^{(2)}(t) \equiv(T-t) \lim _{x_{i} \downarrow B_{i}(t)} F_{i}^{(2)}\left(t, x_{i}\right) \\
& =\mathrm{N}^{\prime}\left(d_{i}\right) \frac{2\left(p_{i}-1\right)}{\sigma_{i}} \sqrt{T-t}-\mathrm{N}\left(d_{i}\right) p_{i}\left(p_{i}-1\right)(T-t), \\
& h_{i}\left(s, x_{i}\right)=\frac{\ln \frac{x_{i}}{K_{i}}-\eta_{i} t}{\sigma_{i} \sqrt{2 \pi(s-t)^{3}}} \exp \left\{-\frac{\left[\ln \frac{x_{i}}{K_{i}}-\eta_{i} t-\frac{p_{i} \sigma_{i}^{2}}{2}(s-t)\right]^{2}}{2 \sigma_{i}^{2}(s-t)}\right\} \text {, } \\
& d_{i}=\frac{\left(r-\eta_{i}-\sigma_{i}^{2} / 2\right)(T-t)}{\sigma_{i} \sqrt{T-t}}=-\frac{p_{i} \sigma_{i}}{2} \sqrt{T-t},
\end{aligned}
$$

with $\mathrm{N}^{\prime}(\cdot)$ being the standard normal density function, and $\sigma_{i} \equiv \sigma_{i}(z)$ when no possible confusion is caused.

b) $w_{i}^{(3)}$ is given by

$$
w_{i}^{(3)}\left(t, x_{i}\right)=\hat{w}_{i}^{(3)}\left(t, x_{i}\right)-(T-t) F_{i}^{(3)}\left(t, x_{i}\right),
$$

where

$$
F_{i}^{(3)}\left(t, x_{i}\right) \equiv x_{i} \frac{\partial}{\partial x_{i}}\left(x_{i}^{2} \frac{\partial^{2} Q_{i}}{\partial x_{i}^{2}}\right)
$$




$$
\begin{aligned}
= & \mathrm{N}^{\prime}\left(d_{2(i)}^{+}\right)\left[\frac{\left(d_{2(i)}^{+}\right)^{2}-1}{\sigma_{i}^{3}(T-t)^{3 / 2}}+\frac{d_{2(i)}^{+}}{\sigma_{i}^{2}(T-t)}\right]+\mathrm{N}\left(d_{2(i)}^{-}\right) p_{i}^{2}\left(1-p_{i}\right)\left(\frac{x_{i}}{B_{i}(t)}\right)^{p_{i}} \\
& +\mathrm{N}^{\prime}\left(d_{2(i)}^{-}\right)\left[\frac{\left(d_{2(i)}^{-}\right)^{2}-1}{\sigma_{i}^{3}(T-t)^{3 / 2}}+\frac{d_{2(i)}^{-}\left(3 p_{i}-1\right)}{\sigma_{i}^{2}(T-t)}+\frac{p_{i}\left(3 p_{i}-2\right)}{\sigma_{i} \sqrt{T-t}}\right]\left(\frac{x_{i}}{B_{i}(t)}\right)^{p_{i}}, \\
\hat{w}_{i}^{(3)}\left(t, x_{i}\right)= & \int_{t}^{T} g_{i}^{(3)}(s) h_{i}\left(s, x_{i}\right) \mathrm{d} s, \\
g_{i}^{(3)}(t) \equiv & (T-t) \lim _{x_{i} \downarrow B_{i}(t)} F_{i}^{(3)}\left(t, x_{i}\right) \\
= & -\mathrm{N}^{\prime}\left(d_{i}\right) \frac{1}{\sigma_{i}^{3}}\left(\frac{2}{\sqrt{T-t}}+4 p_{i} r \sqrt{T-t}\right)+\mathrm{N}\left(d_{i}\right) p_{i}^{2}\left(1-p_{i}\right)(T-t) .
\end{aligned}
$$

c) $w_{i}^{(0)}$ is given by

$$
w_{i}^{(0)}\left(t, x_{i}\right)=\hat{w}_{i}^{(0)}\left(t, x_{i}\right)+(T-t) F_{i}^{(0)}\left(t, x_{i}\right)-\frac{1}{2}(T-t)^{2} \sigma_{i} F_{i}^{(2)}\left(t, x_{i}\right),
$$

where

$$
\begin{aligned}
F_{i}^{(0)}\left(t, x_{i}\right) \equiv & \frac{\partial Q_{i}}{\partial \sigma_{i}} \\
= & \mathrm{N}^{\prime}\left(d_{2(i)}^{+}\right)\left[-\frac{d_{2(i)}^{+}}{\sigma_{i}}-\sqrt{T-t}\right]+\mathrm{N}^{\prime}\left(d_{2(i)}^{-}\right)\left[\frac{d_{2(i)}^{-}}{\sigma_{i}}+\sqrt{T-t}\right]\left(\frac{x_{i}}{B_{i}(t)}\right)^{p_{i}} \\
& +\mathrm{N}\left(d_{2(i)}^{-}\right)\left[\frac{2}{\sigma_{i}}\left(p_{i}-1\right) \ln \left(\frac{x_{i}}{B_{i}(t)}\right)\right]\left(\frac{x_{i}}{B_{i}(t)}\right)^{p_{i}}, \\
\hat{w}_{i}^{(0)}\left(t, x_{i}\right)= & \int_{t}^{T} g_{i}^{(0)}(s) h_{i}\left(s, x_{i}\right) \mathrm{d} s, \\
g_{i}^{(0)}(t) \equiv & -(T-t) \lim _{x_{i} \downarrow B_{i}(t)} F_{i}^{(0)}\left(t, x_{i}\right)+\frac{1}{2}(T-t)^{2} \sigma_{i} \lim _{x_{i} \downarrow B_{i}(t)} F_{i}^{(2)}\left(t, x_{i}\right) \\
= & \mathrm{N}^{\prime}\left(d_{i}\right)\left(p_{i}-1\right)(T-t)^{3 / 2}-\mathrm{N}\left(d_{i}\right) \frac{\sigma_{i}}{2} p_{i}\left(p_{i}-1\right)(T-t)^{2} .
\end{aligned}
$$

d) $w_{i}^{(1)}$ is given by

$$
w_{i}^{(1)}\left(t, x_{i}\right)=\hat{w}_{i}^{(1)}\left(t, x_{i}\right)+(T-t) F_{i}^{(1)}\left(t, x_{i}\right)-\frac{1}{2}(T-t)^{2} \sigma_{i} F_{i}^{(3)}\left(t, x_{i}\right),
$$

where

$$
\begin{aligned}
F_{i}^{(1)}\left(t, x_{i}\right) & \equiv x_{i} \frac{\partial}{\partial x_{i}}\left(\frac{\partial Q_{i}}{\partial \sigma_{i}}\right) \\
& =\mathrm{N}^{\prime}\left(d_{2(i)}^{+}\right)\left[\frac{\left(d_{2(i)}^{+}\right)^{2}-1}{\sigma_{i}^{2} \sqrt{T-t}}+\frac{d_{2(i)}^{+}}{\sigma_{i}}\right]+\mathrm{N}^{\prime}\left(d_{2(i)}^{-}\right)\left[\frac{\left(d_{2(i)}^{-}\right)^{2}-1}{\sigma_{i}^{2} \sqrt{T-t}}\right.
\end{aligned}
$$




$$
\begin{aligned}
& \left.+\left(p_{i}+1\right) \frac{d_{2(i)}^{-}}{\sigma_{i}}+p_{i} \sqrt{T-t}+\frac{2\left(1-p_{i}\right)}{\sigma_{i}^{2} \sqrt{T-t}} \ln \left(\frac{x_{i}}{B_{i}(t)}\right)\right]\left(\frac{x_{i}}{B_{i}(t)}\right)^{p_{i}} \\
& +\mathrm{N}\left(d_{2(i)}^{-}\right)\left[\frac{2}{\sigma_{i}}\left(p_{i}-1\right)\left(1+p_{i} \ln \left(\frac{x_{i}}{B_{i}(t)}\right)\right)\right]\left(\frac{x_{i}}{B_{i}(t)}\right)^{p_{i}}, \\
\hat{w}_{i}^{(1)}\left(t, x_{i}\right)= & \int_{t}^{T} g_{i}^{(1)}(s) h_{i}\left(s, x_{i}\right) \mathrm{d} s, \\
g_{i}^{(1)}(t) \equiv & -(T-t) \lim _{x_{i} \downarrow B_{i}(t)} F_{i}^{(1)}\left(t, x_{i}\right)+\frac{1}{2}(T-t)^{2} \sigma_{i} \lim _{x_{i} \downarrow B_{i}(t)} F_{i}^{(3)}\left(t, x_{i}\right) \\
= & \mathrm{N}^{\prime}\left(d_{i}\right) \frac{1}{\sigma_{i}^{2}}\left[\sqrt{T-t}-2 p_{i} r(T-t)^{3 / 2}\right] \\
& -\mathrm{N}\left(d_{i}\right)\left[\frac{2}{\sigma_{i}}\left(p_{i}-1\right)(T-t)+\frac{\sigma_{i}}{2} p_{i}^{2}\left(p_{i}-1\right)(T-t)^{2}\right] .
\end{aligned}
$$

Proof We use the same technique as in Fouque, Sircar and Solna (2005), but here we need to deal with the time varying hitting boundary $B_{i}(t)$, whereas they considered a constant hitting boundary.

a) If we define

$$
\mathcal{L}_{2}^{(i)} \equiv \frac{\partial}{\partial t}+\frac{1}{2} f_{i}^{2}(y, z) x_{i}^{2} \frac{\partial^{2}}{\partial x_{i}^{2}}+r x_{i} \frac{\partial}{\partial x_{i}}
$$

then $\left\langle\mathcal{L}_{2}^{(i)}\right\rangle x_{i}^{k} \frac{\partial^{k} Q_{i}}{\partial x_{i}^{k}}=0$ for $k \geq 1$, where we used the fact that $\left\langle\mathcal{L}_{2}^{(i)}\right\rangle$ commutes with $x_{i}^{k} \frac{\partial^{k}}{\partial x_{i}^{k}}$ and the fact that $\left\langle\mathcal{L}_{2}^{(i)}\right\rangle Q_{i}=0$. Now we define

$$
\hat{w}_{i}^{(2)} \equiv w_{i}^{(2)}+(T-t) x_{i}^{2} \frac{\partial^{2} Q_{i}}{\partial x_{i}^{2}}
$$

which satisfies

$$
\begin{aligned}
\left\langle\mathcal{L}_{2}^{(i)}\right\rangle \hat{w}_{i}^{(2)}\left(t, x_{i}\right) & =0, \quad x_{i}>B_{i}(t), t<T, \\
\hat{w}_{i}^{(2)}\left(t, B_{i}(t)\right) & =(T-t) \lim _{x_{i} \downarrow B_{i}(t)} x_{i}^{2} \frac{\partial^{2} Q_{i}}{\partial x_{i}^{2}} \equiv g_{i}^{(2)}(t), \quad t \leq T, \\
\hat{w}_{i}^{(2)}\left(T, x_{i}\right) & =0, \quad x_{i}>B_{i}(t) .
\end{aligned}
$$

Change of variables: define

$$
\xi_{i} \equiv \frac{1}{\sigma_{i}} \ln \frac{x_{i}}{B_{i}(t)}, \quad \bar{\mu}_{i}=\frac{r-\eta_{i}-\sigma_{i}^{2} / 2}{\sigma_{i}}=-\frac{p_{i} \sigma_{i}}{2}, \quad \bar{w}_{i}^{(2)}\left(t, \xi_{i}\right) \equiv \hat{w}_{i}^{(2)}\left(t, x_{i}\right) .
$$

Then $\bar{w}_{i}^{(2)}\left(t, \xi_{i}\right)$ satisfies

$$
\begin{aligned}
\frac{\partial \bar{w}_{i}^{(2)}}{\partial t}+\frac{1}{2} \frac{\partial^{2} \bar{w}_{i}^{(2)}}{\partial \xi_{i}^{2}}+\bar{\mu}_{i} \frac{\partial \bar{w}_{i}^{(2)}}{\partial \xi_{i}} & =0, \quad \xi_{i}>0, t<T, \\
\bar{w}_{i}^{(2)}(t, 0) & =g_{i}^{(2)}(t), \quad t \leq T, \\
\bar{w}_{i}^{(2)}\left(T, \xi_{i}\right) & =0, \quad \xi_{i}>0 .
\end{aligned}
$$


It follows that, see, for example, Karatzas and Shreve (1991), $\bar{w}_{i}^{(2)}$ admits the probabilistic representation:

$$
\bar{w}_{i}^{(2)}\left(t, \xi_{i}\right)=\mathbb{E}^{\mathbb{Q}}\left[g_{i}^{(2)}\left(\tau^{*}\right) \mathbf{1}_{\left\{\tau^{*} \leq T\right\}} \mid \xi_{t}^{(i)}=\xi_{i}\right],
$$

where $\xi^{(i)}$ is a Brownian motion with drift $\bar{\mu}_{i}$ under probability measure $\mathbb{Q}$, and $\tau^{*}$ is the first time that $\xi^{(i)}$ hits 0. By plugging in the distribution of $\tau^{*}$, which is well known and can be found, for example, in Borodin and Salminen (2002), we obtain

$$
\begin{aligned}
\bar{w}_{i}^{(2)}\left(t, \xi_{i}\right) & =\int_{t}^{T} g_{i}^{(2)}(s) \frac{\xi_{i}}{\sqrt{2 \pi(s-t)^{3}}} \exp \left\{-\frac{\left[\xi_{i}+\bar{\mu}_{i}(s-t)\right]^{2}}{2(s-t)}\right\} \mathrm{d} s \\
& =\int_{t}^{T} g_{i}^{(2)}(s) h_{i}(s) \mathrm{d} s .
\end{aligned}
$$

So in summary,

$$
w_{i}^{(2)}=\hat{w}_{i}^{(2)}-(T-t) x_{i}^{2} \frac{\partial^{2} Q_{i}}{\partial x_{i}^{2}}=\hat{w}_{i}^{(2)}-(T-t) F_{i}^{(2)}\left(t, x_{i}\right)
$$

b) The same as that for part a).

c) Note that

$$
\left\langle\mathcal{L}_{2}^{(i)}\right\rangle \frac{\partial Q_{i}}{\partial \sigma_{i}}=-\sigma_{i} x_{i}^{2} \frac{\partial^{2} Q_{i}}{\partial x_{i}^{2}}
$$

which can be obtained by taking derivative with respect to $\sigma_{i}$ on equation $\left\langle\mathcal{L}_{2}^{(i)}\right\rangle Q_{i}=0$. Now define

$$
\hat{w}_{i}^{(0)}=w_{i}^{(0)}-(T-t) \frac{\partial Q_{i}}{\partial \sigma_{i}}+\frac{1}{2}(T-t)^{2} \sigma_{i} x_{i}^{2} \frac{\partial^{2} Q_{i}}{\partial x_{i}^{2}},
$$

then $\hat{w}_{i}^{(0)}\left(t, x_{i}\right)$ satisfies

$$
\begin{aligned}
\left\langle\mathcal{L}_{2}^{(i)}\right\rangle \hat{w}_{i}^{(0)}\left(t, x_{i}\right) & =0, \quad x_{i}>B_{i}(t), t<T, \\
\hat{w}_{i}^{(0)}\left(t, B_{i}(t)\right) & =g_{i}^{(0)}(t), \quad t \leq T, \\
\hat{w}_{i}^{(0)}\left(T, x_{i}\right) & =0, \quad x_{i}>B_{i}(t) .
\end{aligned}
$$

The rest of the proof is as in part a).

d) The same as that for part c), using the fact that

$$
\left\langle\mathcal{L}_{2}^{(i)}\right\rangle x_{i} \frac{\partial}{\partial x_{i}}\left(\frac{\partial Q_{i}}{\partial \sigma_{i}}\right)=-\sigma_{i} x_{i} \frac{\partial}{\partial x_{i}}\left(x_{i}^{2} \frac{\partial^{2} Q_{i}}{\partial x_{i}^{2}}\right)
$$

All the calculations of the formulas within this lemma are straightforward. The proof is complete. 
Lemma 3 For each pair $(i, j) \in\{1,2, \cdots, n\} \times\{1,2, \cdots, n\}$ with $i \neq j$ :

a) $w_{i j}^{(3)}$ is given by

$$
w_{i j}^{(3)}\left(t, x_{i}, x_{j}\right)=\hat{w}_{i j}^{(3)}\left(t, x_{i}, x_{j}\right)-(T-t) F_{i j}^{(3)}\left(t, x_{i}, x_{j}\right),
$$

where

$$
\begin{aligned}
& F_{i j}^{(3)}\left(t, x_{i}, x_{j}\right) \equiv\left(x_{i} \frac{\partial Q_{i}}{\partial x_{i}}\right)\left(x_{j}^{2} \frac{\partial^{2} Q_{j}}{\partial x_{j}^{2}}\right) \\
& =F_{j}^{(2)}\left(t, x_{j}\right)\left[\mathrm{N}^{\prime}\left(d_{2(i)}^{+}\right) \frac{1}{\sigma_{i} \sqrt{T-t}}\right. \\
& \left.+\mathrm{N}^{\prime}\left(d_{2(i)}^{-}\right) \frac{1}{\sigma_{i} \sqrt{T-t}}\left(\frac{x_{i}}{B_{i}(t)}\right)^{p_{i}}-\mathrm{N}\left(d_{2(i)}^{-}\right) p_{i}\left(\frac{x_{i}}{B_{i}(t)}\right)^{p_{i}}\right], \\
& \hat{w}_{i j}^{(3)}\left(t, x_{i}, x_{j}\right)=\int_{t}^{T} \int_{0}^{\infty} \bar{G}_{i j}^{(j)}(s, \xi) \psi_{j}\left(s, \xi, x_{j}\right) h_{i j}\left(s, x_{i}, x_{j}\right) \mathrm{d} \xi \mathrm{d} s \\
& +\int_{t}^{T} \int_{0}^{\infty} \bar{G}_{i j}^{(i)}(s, \xi) \psi_{i}\left(s, \xi, x_{i}\right) h_{j i}\left(s, x_{j}, x_{i}\right) \mathrm{d} \xi \mathrm{d} s \\
& \psi_{j}\left(s, \xi, x_{j}\right)=\frac{2}{\sqrt{2 \pi(s-t)}} \sinh \left[\frac{\xi \ln \frac{x_{j}}{B_{j}(t)}}{\sigma_{j}(s-t)}\right] \exp \left\{-\frac{\xi^{2}}{2(s-t)}-\frac{p_{j} \sigma_{j}}{2} \xi\right\}, \\
& h_{i j}\left(s, x_{i}, x_{j}\right)=h_{i}\left(s, x_{i}\right) \exp \left\{-\frac{\left[\ln \frac{x_{j}}{K_{j}}-\eta_{j} t-\frac{p_{j} \sigma_{j}^{2}}{2}(s-t)\right]^{2}}{2 \sigma_{j}^{2}(s-t)}\right\}, \\
& \bar{G}_{i j}^{(j)}(t, \xi) \equiv(T-t) F_{i j}^{(3)}\left(t, B_{i}(t), B_{j}(t) \mathrm{e}^{\sigma_{j} \xi}\right) \\
& =\left[\mathrm{N}^{\prime}\left(d_{i}\right) \frac{2}{\sigma_{i}} \sqrt{T-t}-\mathrm{N}\left(d_{i}\right) p_{i}(T-t)\right] \times \\
& \left\{\mathrm{N}^{\prime}\left(\bar{d}_{2(j)}^{+}\right)\left[-\frac{\bar{d}_{2(j)}^{+}}{\sigma_{j}^{2}(T-t)}-\frac{1}{\sigma_{j} \sqrt{T-t}}\right]-\mathrm{N}\left(\bar{d}_{2(j)}^{-}\right) p_{j}\left(p_{j}-1\right) \mathrm{e}^{p_{j} \sigma_{j} \xi}\right. \\
& \left.+\mathrm{N}^{\prime}\left(\bar{d}_{2(j)}^{-}\right)\left[\frac{\bar{d}_{2(j)}^{-}}{\sigma_{j}^{2}(T-t)}+\frac{2 p_{j}-1}{\sigma_{j} \sqrt{T-t}}\right] \mathrm{e}^{p_{j} \sigma_{j} \xi}\right\} \\
& \bar{G}_{i j}^{(i)}(t, \xi) \equiv(T-t) F_{i j}^{(3)}\left(t, B_{i}(t) \mathrm{e}^{\sigma_{i} \xi}, B_{j}(t)\right) \\
& =\left[\mathrm{N}^{\prime}\left(d_{j}\right) \frac{2\left(p_{j}-1\right)}{\sigma_{j}} \sqrt{T-t}-\mathrm{N}\left(d_{j}\right) p_{j}\left(p_{j}-1\right)(T-t)\right] \times \\
& {\left[\mathrm{N}^{\prime}\left(\bar{d}_{2(i)}^{+}\right) \frac{1}{\sigma_{i} \sqrt{T-t}}+\mathrm{N}^{\prime}\left(\bar{d}_{2(i)}^{-}\right) \frac{\mathrm{e}^{p_{i} \sigma_{i} \xi}}{\sigma_{i} \sqrt{T-t}}-\mathrm{N}\left(\bar{d}_{2(i)}^{-}\right) p_{i} \mathrm{e}^{p_{i} \sigma_{i} \xi}\right],} \\
& \bar{d}_{2(i)}^{ \pm}= \pm \xi-\frac{p_{i} \sigma_{i}}{2} \sqrt{T-t} .
\end{aligned}
$$

b) $w_{i j}^{(1)}$ is given by

$$
w_{i j}^{(1)}\left(t, x_{i}, x_{j}\right)=\hat{w}_{i j}^{(1)}\left(t, x_{i}, x_{j}\right)+(T-t) F_{i j}^{(1)}\left(t, x_{i}, x_{j}\right)-\frac{1}{2}(T-t)^{2} \sigma_{j} F_{i j}^{(3)}\left(t, x_{i}, x_{j}\right),
$$


where

$$
\begin{aligned}
& F_{i j}^{(1)}\left(t, x_{i}, x_{j}\right) \equiv\left(x_{i} \frac{\partial Q_{i}}{\partial x_{i}}\right)\left(\frac{\partial Q_{j}}{\partial \sigma_{j}}\right) \\
& =F_{j}^{(0)}\left(t, x_{j}\right)\left[\mathrm{N}^{\prime}\left(d_{2(i)}^{+}\right) \frac{1}{\sigma_{i} \sqrt{T-t}}\right. \\
& \left.+\mathrm{N}^{\prime}\left(d_{2(i)}^{-}\right) \frac{1}{\sigma_{i} \sqrt{T-t}}\left(\frac{x_{i}}{B_{i}(t)}\right)^{p_{i}}-\mathrm{N}\left(d_{2(i)}^{-}\right) p_{i}\left(\frac{x_{i}}{B_{i}(t)}\right)^{p_{i}}\right], \\
& \hat{w}_{i j}^{(1)}\left(t, x_{i}, x_{j}\right)=\int_{t}^{T} \int_{0}^{\infty} \bar{H}_{i j}^{(j)}(s, \xi) \psi_{j}\left(s, \xi, x_{j}\right) h_{i j}\left(s, x_{i}, x_{j}\right) \mathrm{d} \xi \mathrm{d} s \\
& +\int_{t}^{T} \int_{0}^{\infty} \bar{H}_{i j}^{(i)}(s, \xi) \psi_{i}\left(s, \xi, x_{i}\right) h_{j i}\left(s, x_{j}, x_{i}\right) \mathrm{d} \xi \mathrm{d} s, \\
& \bar{H}_{i j}^{(j)}(t, \xi) \equiv-(T-t) F_{i j}^{(1)}\left(t, B_{i}(t), B_{j} \mathrm{e}^{\sigma_{j} \xi}\right)+\frac{1}{2}(T-t)^{2} \sigma_{j} F_{i j}^{(3)}\left(t, B_{i}(t), B_{j} \mathrm{e}^{\sigma_{j} \xi}\right) \\
& =\left[\mathrm{N}^{\prime}\left(d_{i}\right) \frac{2}{\sigma_{i}} \sqrt{T-t}-\mathrm{N}\left(d_{i}\right) p_{i}(T-t)\right] \times \\
& \left\{\mathrm{N}^{\prime}\left(\bar{d}_{2(j)}^{+}\right)\left[\frac{d_{2(j)}^{+}}{2 \sigma_{j}}+\frac{1}{2} \sqrt{T-t}\right]+\mathrm{N}^{\prime}\left(\bar{d}_{2(j)}^{-}\right)\left[-\frac{\bar{d}_{2(j)}^{-}}{2 \sigma_{j}}+\left(p_{j}-\frac{3}{2}\right) \sqrt{T-t}\right] \mathrm{e}^{p_{j} \sigma_{j} \xi}\right. \\
& \left.-\mathrm{N}\left(\bar{d}_{2(j)}^{-}\right)\left(p_{j}-1\right)\left[2 \xi+\frac{p_{j} \sigma_{j}}{2}(T-t)\right] \mathrm{e}^{p_{j} \sigma_{j} \xi}\right\} \\
& \bar{H}_{i j}^{(i)}(t, \xi) \equiv-(T-t) F_{i j}^{(1)}\left(t, B_{i}(t) \mathrm{e}^{\sigma_{i} \xi}, B_{j}(t)\right)+\frac{1}{2}(T-t)^{2} \sigma_{j} F_{i j}^{(3)}\left(t, B_{i}(t) \mathrm{e}^{\sigma_{i} \xi}, B_{j}(t)\right) \\
& =\left[\mathrm{N}^{\prime}\left(d_{j}\right)\left(p_{j}-1\right)(T-t)^{3 / 2}-\mathrm{N}\left(d_{j}\right) \frac{p_{j} \sigma_{j}}{2}\left(p_{j}-1\right)(T-t)^{2}\right] \times \\
& {\left[\mathrm{N}^{\prime}\left(\bar{d}_{2(i)}^{+}\right) \frac{1}{\sigma_{i} \sqrt{T-t}}+\mathrm{N}^{\prime}\left(\bar{d}_{2(i)}^{-}\right) \frac{\mathrm{e}^{p_{i} \sigma_{i} \xi}}{\sigma_{i} \sqrt{T-t}}-\mathrm{N}\left(\bar{d}_{2(i)}^{-}\right) p_{i} \mathrm{e}^{p_{i} \sigma_{i} \xi}\right] .}
\end{aligned}
$$

c) $w_{i j}^{(4)}$ is given by

$$
w_{i j}^{(4)}\left(t, x_{i}, x_{j}\right)=\hat{w}_{i j}^{(4)}\left(t, x_{i}, x_{j}\right)-(T-t) F_{i j}^{(4)}\left(t, x_{i}, x_{j}\right),
$$

where

$$
\begin{aligned}
F_{i j}^{(4)}\left(t, x_{i}, x_{j}\right)= & -\left(x_{i} \frac{\partial Q_{i}}{\partial x_{i}}\right)\left(x_{j} \frac{\partial Q_{j}}{\partial x_{j}}\right) \\
= & -\left[\mathrm{N}^{\prime}\left(d_{2(i)}^{+}\right) \frac{1}{\sigma_{i} \sqrt{T-t}}+\mathrm{N}^{\prime}\left(d_{2(i)}^{-}\right) \frac{1}{\sigma_{i} \sqrt{T-t}}\left(\frac{x_{i}}{B_{i}(t)}\right)^{p_{i}}\right. \\
& \left.\quad-\mathrm{N}\left(d_{2(i)}^{-}\right) p_{i}\left(\frac{x_{i}}{B_{i}(t)}\right)^{p_{i}}\right]\left[-\mathrm{N}\left(d_{2(j)}^{-}\right) p_{j}\left(\frac{x_{j}}{B_{j}(t)}\right)^{p_{j}}\right. \\
& \left.+\mathrm{N}^{\prime}\left(d_{2(j)}^{+}\right) \frac{1}{\sigma_{j} \sqrt{T-t}}+\mathrm{N}^{\prime}\left(d_{2(j)}^{-}\right) \frac{1}{\sigma_{j} \sqrt{T-t}}\left(\frac{x_{j}}{B_{j}(t)}\right)^{p_{j}}\right], \\
\hat{w}_{i j}^{(4)}\left(t, x_{i}, x_{j}\right)= & \int_{t}^{T} \int_{0}^{\infty} \bar{K}_{i j}^{(j)}(s, \xi) \psi_{j}\left(s, \xi, x_{j}\right) h_{i j}\left(s, x_{i}, x_{j}\right) \mathrm{d} \xi \mathrm{d} s \\
& +\int_{t}^{T} \int_{0}^{\infty} \bar{K}_{i j}^{(i)}(s, \xi) \psi_{i}\left(s, \xi, x_{i}\right) h_{j i}\left(s, x_{j}, x_{i}\right) \mathrm{d} \xi \mathrm{d} s,
\end{aligned}
$$




$$
\begin{aligned}
\bar{K}_{i j}^{(j)}(t, \xi) & \equiv(T-t) F_{i j}^{(4)}\left(t, B_{i}(t), B_{j}(t) \mathrm{e}^{\sigma_{j} \xi}\right), \\
\bar{K}_{i j}^{(i)}(t, \xi) & \equiv(T-t) F_{i j}^{(4)}\left(t, B_{i}(t) \mathrm{e}^{\sigma_{i} \xi}, B_{j}(t)\right) .
\end{aligned}
$$

Proof

a) If we define

$$
\mathcal{L}_{2}^{(i j)} \equiv \frac{\partial}{\partial t}+\frac{1}{2} f_{i}^{2}(y, z) x_{i}^{2} \frac{\partial^{2}}{\partial x_{i}^{2}}+\frac{1}{2} f_{j}^{2}(y, z) x_{j}^{2} \frac{\partial^{2}}{\partial x_{j}^{2}}+r x_{i} \frac{\partial}{\partial x_{i}}+r x_{j} \frac{\partial}{\partial x_{j}},
$$

then $\left\langle\mathcal{L}_{2}^{(i j)}\right\rangle x_{i}^{k} \frac{\partial^{k} Q_{i}}{\partial x_{i}^{k}}=0$ for $k \geq 1$. Next we define

$$
\hat{w}_{i j}^{(3)} \equiv w_{i j}^{(3)}+(T-t)\left(x_{i} \frac{\partial Q_{i}}{\partial x_{i}}\right)\left(x_{j}^{2} \frac{\partial^{2} Q_{j}}{\partial x_{j}^{2}}\right)
$$

which satisfies

$$
\begin{aligned}
\left\langle\mathcal{L}_{2}^{(i j)}\right\rangle \hat{w}_{i j}^{(3)}\left(t, x_{i}, x_{j}\right) & =0, \quad x_{i}>B_{i}(t), x_{j}>B_{j}(t), t<T, \\
\hat{w}_{i j}^{(3)}\left(t, B_{i}(t), x_{j}\right) & =(T-t) F_{i j}^{(3)}\left(t, B_{i}(t), x_{j}\right) \equiv G_{i j}^{(j)}\left(t, x_{j}\right), \quad x_{j}>B_{j}(t), t \leq T, \\
\hat{w}_{i j}^{(3)}\left(t, x_{i}, B_{j}(t)\right) & =(T-t) F_{i j}^{(3)}\left(t, x_{i}, B_{j}(t)\right) \equiv G_{i j}^{(i)}\left(t, x_{i}\right), \quad x_{i}>B_{i}(t), t \leq T, \\
\hat{w}_{i j}^{(3)}\left(T, x_{i}, x_{j}\right) & =0, \quad x_{i}>B_{i}(t), x_{j}>B_{j}(t) .
\end{aligned}
$$

Change of variables: define

$$
\begin{array}{cc}
\xi_{i} \equiv \frac{1}{\sigma_{i}} \ln \frac{x_{i}}{B_{i}(t)}, & \xi_{j} \equiv \frac{1}{\sigma_{j}} \ln \frac{x_{j}}{B_{j}(t)}, \\
\bar{\mu}_{i} \equiv \frac{r-\eta_{i}-\sigma_{i}^{2} / 2}{\sigma_{i}}=-\frac{p_{i} \sigma_{i}}{2}, & \bar{\mu}_{j} \equiv \frac{r-\eta_{j}-\sigma_{j}^{2} / 2}{\sigma_{j}}=-\frac{p_{j} \sigma_{j}}{2},
\end{array}
$$

and also

$$
\begin{aligned}
\bar{w}_{i j}^{(3)}\left(t, \xi_{i}, \xi_{j}\right) & \equiv \hat{w}_{i j}^{(3)}\left(t, x_{i}, x_{j}\right) \\
\bar{G}_{i j}^{(j)}\left(t, \xi_{j}\right) & \equiv G_{i j}^{(j)}\left(t, x_{j}\right)=G_{i j}^{(j)}\left(t, B_{j}(t) \mathrm{e}^{\sigma_{j} \xi_{j}}\right), \\
\bar{G}_{i j}^{(i)}\left(t, \xi_{i}\right) & \equiv G_{i j}^{(i)}\left(t, x_{i}\right)=G_{i j}^{(i)}\left(t, B_{i}(t) \mathrm{e}^{\sigma_{i} \xi_{i}}\right) .
\end{aligned}
$$

Then we have

$$
\begin{aligned}
\frac{\partial \bar{w}_{i j}^{(3)}}{\partial t}+\frac{1}{2} \frac{\partial^{2} \bar{w}_{i j}^{(3)}}{\partial \xi_{i}^{2}}+\frac{1}{2} \frac{\partial^{2} \bar{w}_{i j}^{(3)}}{\partial \xi_{j}^{2}}+\bar{\mu}_{i} \frac{\partial \bar{w}_{i j}^{(3)}}{\partial \xi_{i}}+\bar{\mu}_{j} \frac{\partial \bar{w}_{i j}^{(3)}}{\partial \xi_{j}}=0, & \xi_{i}>0, \xi_{j}>0, t<T, \\
\bar{w}_{i j}^{(3)}\left(t, 0, \xi_{j}\right)=\bar{G}_{i j}^{(j)}\left(t, \xi_{j}\right), & \xi_{j}>0, t \leq T, \\
\bar{w}_{i j}^{(3)}\left(t, \xi_{i}, 0\right)=\bar{G}_{i j}^{(i)}\left(t, \xi_{i}\right), & \xi_{i}>0, t \leq T, \\
\bar{w}_{i j}^{(3)}\left(T, \xi_{i}, \xi_{j}\right)=0, & \xi_{i}>0, \xi_{j}>0, t<T .
\end{aligned}
$$


The function $\bar{w}_{i j}^{(3)}$ admits the probabilistic representation:

$$
\begin{aligned}
\bar{w}_{i j}^{(3)}\left(t, \xi_{i}, \xi_{j}\right)= & \mathbb{E}^{\mathbb{Q}}\left\{\bar{G}_{i j}^{(j)}\left(\bar{\tau}, \xi_{\bar{\tau}}^{(j)}\right) \mathbf{1}_{\left\{\bar{\tau}=\bar{\tau}_{i}\right\}} \mathbf{1}_{\{\bar{\tau} \leq T\}}\right. \\
& \left.+\bar{G}_{i j}^{(i)}\left(\bar{\tau}, \xi_{\bar{\tau}}^{(i)}\right) \mathbf{1}_{\left\{\bar{\tau}=\bar{\tau}_{j}\right\}} \mathbf{1}_{\{\bar{\tau} \leq T\}} \mid \xi_{t}^{(i)}=\xi_{i}, \xi_{t}^{(j)}=\xi_{j}\right\},
\end{aligned}
$$

where $\xi^{(i)}$ and $\xi^{(j)}$ are independent Brownian motions with drifts $\bar{\mu}_{i}$ and $\bar{\mu}_{j}$, respectively, under probability measure $\mathbb{Q}, \bar{\tau}_{i}\left(\bar{\tau}_{j}\right)$ is the first time that $\xi^{(i)}\left(\xi^{(j)}\right)$ hits 0 , and $\bar{\tau}=\min \left\{\bar{\tau}_{i}, \bar{\tau}_{j}\right\}$. From Borodin and Salminen (2002), we have

$$
\begin{aligned}
\bar{h}_{i j}\left(s, \xi ; \xi_{i}, \xi_{j}\right) \equiv & \mathbb{Q}\left\{\bar{\tau} \in \mathrm{d} s, \bar{\tau}=\bar{\tau}_{i}, \xi_{\bar{\tau}}^{(j)} \in \mathrm{d} \xi \mid \xi_{t}^{(i)}=\xi_{i}, \xi_{t}^{(j)}=\xi_{j}\right\} \\
= & \frac{2}{\sqrt{2 \pi(s-t)}} \sinh \left(\frac{\xi \xi_{j}}{s-t}\right) \exp \left\{\bar{\mu}_{j} \xi-\frac{\xi^{2}}{2(s-t)}\right\} \exp \left\{-\frac{\left[\xi_{j}+\bar{\mu}_{j}(s-t)\right]^{2}}{2(s-t)}\right\} \\
& \times \frac{\xi_{i}}{\sqrt{2 \pi(s-t)^{3}}} \exp \left\{-\frac{\left[\xi_{i}+\bar{\mu}_{i}(s-t)\right]^{2}}{2(s-t)}\right\} \mathrm{d} \xi \mathrm{d} s .
\end{aligned}
$$

Therefore,

$$
\begin{aligned}
\bar{w}_{i j}^{(3)}\left(t, \xi_{i}, \xi_{j}\right)= & \int_{t}^{T} \int_{0}^{\infty} \bar{G}_{i j}^{(j)}(s, \xi) \bar{h}_{i j}\left(s, \xi ; \xi_{i}, \xi_{j}\right) \mathrm{d} \xi \mathrm{d} s \\
& +\int_{t}^{T} \int_{0}^{\infty} \bar{G}_{i j}^{(i)}(s, \xi) \bar{h}_{j i}\left(s, \xi ; \xi_{j}, \xi_{i}\right) \mathrm{d} \xi \mathrm{d} s .
\end{aligned}
$$

Now change $\left(\xi_{i}, \xi_{j}\right)$ back to $\left(x_{i}, x_{j}\right)$ to obtain the desired result.

b) Define

$$
\hat{w}_{i j}^{(1)} \equiv w_{i j}^{(1)}-(T-t)\left(x_{i} \frac{\partial Q_{i}}{\partial x_{i}}\right)\left(\frac{\partial Q_{j}}{\partial \sigma_{j}}\right)+\frac{1}{2}(T-t)^{2} \sigma_{j}\left(x_{i} \frac{\partial Q_{i}}{\partial x_{i}}\right)\left(x_{j}^{2} \frac{\partial^{2} Q_{j}}{\partial x_{j}^{2}}\right) .
$$

Then $\hat{w}_{i j}^{(1)}$ satisfies

$$
\begin{aligned}
& \left\langle\mathcal{L}_{2}^{(i j)}\right\rangle \hat{w}_{i j}^{(1)}\left(t, x_{i}, x_{j}\right)=0, \quad x_{i}>B_{i}(t), x_{j}>B_{j}(t), t<T, \\
& \hat{w}_{i j}^{(1)}\left(t, B_{i}(t), x_{j}\right)=-(T-t) F_{i j}^{(1)}\left(t, B_{i}(t), x_{j}\right)+\frac{1}{2}(T-t)^{2} \sigma_{j} F_{i j}^{(3)}\left(t, B_{i}(t), x_{j}\right), \\
& x_{j}>B_{j}(t), t \leq T, \\
& \hat{w}_{i j}^{(1)}\left(t, x_{i}, B_{j}(t)\right)=-(T-t) F_{i j}^{(1)}\left(t, x_{i}, B_{j}(t)\right)+\frac{1}{2}(T-t)^{2} \sigma_{j} F_{i j}^{(3)}\left(t, x_{i}, B_{j}(t)\right) \text {, } \\
& x_{i}>B_{i}(t), t \leq T, \\
& \hat{w}_{i j}^{(1)}\left(T, x_{i}, x_{j}\right)=0, \quad x_{i}>B_{i}(t), x_{j}>B_{j}(t) .
\end{aligned}
$$

The rest of the proof is similar to that of part a).

c) Define

$$
\hat{w}_{i j}^{(4)} \equiv w_{i j}^{(4)}-(T-t)\left(x_{i} \frac{\partial Q_{i}}{\partial x_{i}}\right)\left(x_{j} \frac{\partial Q_{j}}{\partial x_{j}}\right) .
$$


Then $\hat{w}_{i j}^{(4)}$ satisfies

$$
\begin{array}{ccc}
\left\langle\mathcal{L}_{2}^{(i j)}\right\rangle \hat{w}_{i j}^{(4)}\left(t, x_{i}, x_{j}\right)= & 0, \quad x_{i}>B_{i}(t), x_{j}>B_{j}(t), t<T, \\
\hat{w}_{i j}^{(1)}\left(t, B_{i}(t), x_{j}\right)= & -(T-t) F_{i j}^{(4)}\left(t, B_{i}(t), x_{j}\right), \\
& x_{j}>B_{j}(t), t \leq T, \\
\hat{w}_{i j}^{(4)}\left(t, x_{i}, B_{j}(t)\right)= & -(T-t) F_{i j}^{(4)}\left(t, x_{i}, B_{j}(t)\right), \\
& x_{i}>B_{i}(t), t \leq T, \\
\hat{w}_{i j}^{(4)}\left(T, x_{i}, x_{j}\right)= & 0, \quad x_{i}>B_{i}(t), x_{j}>B_{j}(t) .
\end{array}
$$

The rest of the proof is similar to that of part a). The proof is complete.

\section{Acknowledgment}

The first author would like to thank Ronnie Sircar and Knut Sølna for the many helpful discussions on the subject. The authors thank an anonymous referee for helpful comments which improved an earlier version of the paper.

\section{References}

[1] F. Black and J. C. Cox (1976). Valuing Corporate Securities: Some Effects of Bond Indenture Provisions. Journal of Finance, 31, 351-367.

[2] A. N. Borodin and P. Salminen (2002). Handbook of Brownian motion: Facts and Formulae, 2nd ed., Birkahauser Verlag.

[3] D. Duffie and K. Singleton (2003) Credit Risk, Princeton University Press.

[4] D. Duffie and N. Garleanu (2001). Risk and Valuation of Collateralized Debt Obligations. Financial Analysts Journal, 57(1) 41-59.

[5] J.P. Fouque, G. Papanicolaou and R. Sircar (2000). Derivatives in Financial Markets with Stochastic Volatility, Cambridge University Press.

[6] J.P. Fouque, G. Papanicolaou, R. Sircar and K. Solna (2003). Multiscale Stochastic Volatility Asymptotics. SIAM Journal on Multiscale Modeling and Simulation, 2(1) 22-42.

[7] J.P. Fouque, R. Sircar and K. Solna (2006). Stochastic Volatility Effects on Defaultable Bonds. Applied Mathematical Finance, 13(3) 215-244.

[8] I. Karatzas and S. Shreve (1991). Brownian Motion and Stochastic Calculus, 2nd ed., SpringerVerlag.

[9] C. Zhou (2001). An Analysis of Default Correlations and Multiple Defaults. Review of Financial Studies, 14(2) 555-576. 